TRAVAIL DE LA CLINIQUE OBSTÉtRIGALE DE LA FACULTÉ DE MÉdeciNe de COPENHAGUE (SERVICE A. RIGSHOSPITAL.)

\title{
Traitement de la syphilis pendant la grossesse, avec étude particulière du sort ultérieur des enfants.
}

\author{
HARALD BOAS \\ Médecin-chef de la policlinique \\ des Maladies cutanées à \\ Copenhague.
}

Par

S. A. GAMMELTOFT.
Professeur à l'Université de co-
et $\begin{gathered}\text { penhague, Accoucheur } \\ \text { en chief. }\end{gathered}$

Au cours des périodes qui se sont écoulées depuis plusieurs dizaines d'années, la lutte contre les maladies infectieuses a été conduite avec une énergie et un succès toujours croissants. Alors mème que les efforts combinés des médecins et des hygiénistes échouent encore quelquefois et qu'une affection contagieuse (comme par exemple, il n'y a pas longtemps encore, la "grippe espagnolen) vient envahir le monde entier avec une irrésistible puissance, cependant on ne peut pas faire autrement que d'ètre rempli d'admiration quand on a vu, par exemple, au cours de la guerre mondiale que le corps médical, aussi bien chez les puissances de l'Entente que dans les Empires centraux, a réussi à mettre un frein à l'expansion de la plupart des maladies contagieuses. Ces maladies n'ont pu ainsi guère dépasser les territoires où elles avaient pris naissance.

Il était tout naturel que les maladies infectieuses qui aient attiré les premières l'attention au cours de la guerre, et à la lutte contre lesquelles on a consacré particulièrement toutes les forces, aient été des fléaux tels que: typhus pétéchial, choléra, peste et fievre typhoïde.

Mais cependant on se relldit bientôt compte qu'il en était d'autres, qui, avec une apparition moins alarmante, exigeaient pour- 
tant qu'on consacrât toutes les forces à les combattre et, sous ce rapport nous devons spécialement citer la tuberculose, la blennorragie et la syphilis.

Sans faire fi des accidents que peuvent provoquer la blennorragie et la tuberculose, on est certainement en droit de considérer la syphilis comme tout particuliérement dangereuse, car, une réviviscence considérable de cette maladie enferme un très sérieux danger pour la génération à venir.

Dans tous les pays dont la population a numériquement souffert de la guerre, on s'est donc rendu compte depuis longtemps cu'il s'agissait d'empècher par tous les moyens qu'on avait à sa disposition, que la réviviscence des maladies vénériennes et tout spécialement de la syphilis (qui suit toujours les grandes guerres) put faire sentir son influence nuisible sur la génération suivante. C'est cette génération que tous les peuples belligérants suivent d'un oeil tout à fait interessé, car, l'avenir de ces nations dépend dans une large mesure de cette génération.

Mais, les pays neutres sont également intéressés à la question. Eux aussi sont exposés; en effet, la syphilis y a pris une plus grande extension qu'auparavant, soit à la suite du relâchement général de la morale qui, depuis les pays belligérants, a gagné largement du terrain, soit aussi parce que ces dernières contrées représentent des foyers depuis lesquels l'infection pout se propager.

Le problème de la forme la plus rationnelle à donner à la lutte contre les affections vénériennes est donc à l'ordre du jour chez tous les peuples civilisés. Cet état d'esprit s'est aussi manifesté dans le Congrès tenu récemment ici à Copenhague et dans lecuel se sont rencontrés des délégués de nombreux pays.

Le point le plus important, c'est naturellement, comme pour toutes les maladies infectieuses, d'empêcher la transmission directe d'individu à individu; mais, pour la syphilis, il s'agit tout particuliérement d'empêcher aussi que la maladie soit déjà transmise in utero à la génération qui va suivre.

Tandis que le premier de ces problèmes est en dehors du cadre de notre travail, nous avons eu, en écrivant celui-ci, l'intention d'étudier de plus près, à l'aide d'ałıondants matériaux, les conditions qui entrent en jeu quand une femme syphilitique devient enceinte et de rechercher quels moyens sont en notre possession pour em- 
pècher que l'enfant ne soit contaminé par la maladie de sa mère.

La question a été déjà soulevée aussi bien par des syphiligraphes (Ödmanson, Matzenauer etc.) que par des accoucheurs (Stangenberg, Reuben Peterson, Lemeland et Brisson, Lobenstine, Schwab, Sauvage, Marie Holth, Loeser, Fullerton, Trinchese, Baisch et beaucoup d'autres encore).

C'est aussi un signe des temps qu'en France aussi, à I'instigation de Pinard et Couvelaire, on ait établi une consultation destinée uniquement aux femmes syphilitiques en état de grossesse. Elle. est rattachée à la cliniçue obstétricale (Clinique Baudelocque) mais le traitement est dirigé par un syphiligraphe de profession. On a établi également des centres semblables en Angleterre (London "Thavies' Imn»: "venereal centre for praegnant women").

Il n'est pourtant pas possible de traiter l'ensemble du problème d'une manière générale; mais pour obtenir un résultat, il est nécessaire de s'occuper plus en détail des diverses circonstances qui peuvent se manifester.

I] sera suffisant d'indiquer brièvement ici quelques-uns des facteurs qui ont de l'importance.

Comme on le démontrera plus loin, il n'est pas indiffèrent qu'il sagisse de cas de syphilis dans lesquels l'infection remonte à plusieurs années ou d'une malade contaminée peu avant, pendant ou après la conception.

De même pour apprécier les résultats, il est important de savoir si la femme en cuestion a été traitée avant on pendant sa grossesse et si, réellement, il en est ainsi, à cuel moment elle a été traitée. cans des matériaux relativement abondants, il est impossible darriver à des résultats tant soit peu certains.

Les matériaux que nous avons cus à notre disposition et au moyen hisquels nous avons essayé de décider quelle est la mailleure maniere de traiter une syphilitique enceinte, comprennent, outre un crtain nombre de cas de la clientèle particulière et de la consultation hopitaliere, toutes les femmes syphilitiques qui, depuis le 1:er janver 1912 jusqu'au 1:er avril 1920, ont accouché dans le service $A$. de I: Maternité du Rigshospital.

Avant de nous occuper d'un peu plus près des résultats et d'ar$\therefore$ ar aux conclusions que nous pensons pouvoir tirer de nos maté2- Ars Gineculogica. Vob. I. Fasc. 3. 
riaux, nous discuterons brièvement les points de vue exposés par d'autres auteurs antérieurs; car, il est également intéressant de considérer les résultats qu'ils ont obtenus. En outre, grâce à nos matériaux, il est possible de se rendre compte jusqu'à quel point, chez les femmes syphilitiques, la grossesse évolue d'une façon différente des femmes normales et, en particulier, de voir si des conditions particulières se manifestent pendant la période de la délivrance et des suites de couches.

Etant domnée la fréquence de la syphilis chez les femmes enceintes, il existe à ce sujet un grand nombre de statistiques.

Cependant toutes les données anciennes, datant de l'époque antérieure à celle où Wassermann fit connaitre sa réaction, sont certainement lépourvues de signification.

Les statistiques datant de périodes ultérieures provicunent en majeure partie des matériaux des grandes cliniques obstétricales.

C'est ainsi que Loeser en 1913, sur 39,806 naissances qui a vaicnt eu lieu dans diverses maternités d'Allemagne, trouva dans 1,5\% des signes de syphilis congénitale chez les enfants.

En 1917, on pratiqua la réaction de Wassermann dans les Maternités de Rostock, Düsseldorf et Munich, sur toutes les parturientes et on constata que $3,9 \%$ des enfants des malades hospitalisées étaient atteintes de syphilis congénitale.

Cependant, il ne ressort pas du travail de Loeser, comment il a posé le diagnostic de syphilis congénitale, s'il s'est contenté d'ume réaction de Wassermann positive chez la mère ou s'il a exigé cette réaction ou des manifestations sypluilitiques chez l'enfant.

En Amérique, nous avons une statistique de Williams qui comprend 10,000 cas. Il constata que 3,5\% des femmes enceintes, exaninées à partir du 7:ème au 10:ème mois de la grossesse, plésentaient des signes de syphilis. Cependant il considère ce chiffre comme un chiffre minimum, entre autres choses, parce que, dans sa statistique, il n'a pas été tenu compte des femmes qui ont accou-

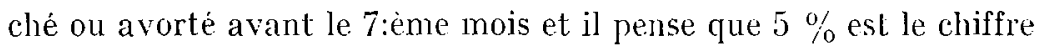
exact.

Williams s'est occupé ultérieurement de cette question et, lors d'une réunion de la Société américaine de gynécologie, en 1920, il déclare qu'il a actuellement examiné en tout 4,000 femnes enceintes, soit blanches, soit de race de couleur, et qu'il a constaté que 
$11,2 \%$ avaient une réaction de Wassermann positive. La différence entre femmes blanches et femmes de couleur est frappante, car il ne trouva que $2,48 \%$ blanches avec réaction positive, tandis que $16,29 \%$ des femmes de couleur donnèrent une réaction de Wassermann positive.

Par contre, Reuben Peterson sur 1,348 cas trouva seulement 44 réactions positives ce qui donne $6,4 \%$.

Goodman a donné un relevé de la Clinique de Sloane.

Sur 1,300 parturientes, il trouva $8,7 \%$ avec réaction de Wassermann positive.

Divers auteurs ont aussi communiqué différentes séries de cas, moins importantes et ce qui frappe le plus, c'est que les résultats varient très considérablement.

Il est difficile évidemment de se faire une idée de la cause de ces grandes variations; elles peuvent tenir aux matériaux eux-mèmes, mais il n'est pas douteux qu'elles reposent aussi en partie sur la façon dont sont exécutées ces réactions de Wassermann.

Nos cas présentent ici le grand avantage que tous les examens du sang sont faits à l'Institut sérologique de l'Etat Danois qui dispose sous ce rapport d'une expérience extrémement considérable et dont le nom est la garantie d'une technique excellente et la plus moderne.

Le problème est tout à fait différent quand il s'agit de savoir $s i$. chez les femmes enceintes et les parturientes, on a le droit d identifier la réaction positive de Wassermann avec la syphilis. Une reaction de Wassermann positive peut ètre due à une confusion ou a une erreur de technique; enfin, une réaction positive chez les enfants, peut ètre due, comme l'ont démontré Thomsen et BoAs, dans des cas très rares, au fait que les substances réagissantes du sarig de la mère sont en circulation dans celui de l'enfant, question sur laquelle nous reviendrons plus tard. Pourtant ce point important ne joue aucun rôle dans nos matériaux. Ce n'est que dans un trés petit nombre de cas qu'une réaction positive isolée nous a fait porter le diagnostic de syphilis. Dans la grande majorité des Gas il a été possible de confirmer le diagnostic de syphilis, soit par un examen plus exact de la malade ou par des recherches cliniques quilus minuticuses. Pour les dernières années en particulier, nous ne nous sommes pas contentés d'une unique réaction de Wassermann 
quand il n'existait pas d'autres points de repère pour le diagnostic; mais toujours, après un laps de temps plus ou moins long. nous avons répété la réaction et nous n'avons pas fait entrer en ligne de compte quelques cas dans lesquels cette deuxième réaction fut négative. Nous croyons donc que le tableau que nous donnons plus loin, et qui fournit une vue d'ensemble sur le nombre des syphilitiques hospitalisées dans le service A de la Maternité de 1912 à 1921, donne une reproduction correcte des conditions réelles.

Si dans le tableau ainsi établi, il devait y avoir quelque erreur, elle consisterait plutôt en ce que le nombre est trop élevé pour les premières années; d'un autre côté, il est également facile de penser qu'il y a eu des syphilitiques latentes avec réaction de Wassermann négative et qui ont échappé à notre attention.

Il ressort nettement du tableau I qu'il y a eu une augmentation au cours des années et, pour 1921, qui du reste, n'est pas compris dans les matériaux cliniques, le chiffre est le plus élevé cqu'on ait encore atteint soit $7,7 \%$.

TABLEAU $I$.

Service A, de la Maternité

\begin{tabular}{|c|c|c|c|c|c|c|c|c|c|c|}
\hline & 1912. & 1913. & 1914. & 1915. & 1916. & 1917 & 1918. & 1919. & 1920 & 1921. \\
\hline $\begin{array}{l}\text { Nombre } \\
\text { des syphiliticques }\end{array}$ & 50 & 65 & 66 & 67 & 70 & 78 & 84 & 74 & 109 & 140 \\
\hline $\begin{array}{l}\text { Nombre } \\
\text { des parturientes }\end{array}$ & 1334 & 1383 & 1401 & $\mid 1442$ & 1572 & 1473 & 1578 & 1536 & 1902 & 1828 \\
\hline Pourcentage & $3,0^{\circ} \%$ & $\left.4,6^{n}\right)$ & $4,7 \%$ & $4,0^{\circ}, 1$ & $4,5^{0}$ & 5,3 ? & $5, \% \%$ & $4,8 \%$ & $5.7 \%$ & $7, x$ \\
\hline
\end{tabular}

On ne peut déterminer avec certitude à quoi est due cette augmentation; elle peut être due au fait que la maladie est plus fréquente qu'autrefois; mais elle peut aussi être due à ce qu'on est davantage sur ses gardes dans le but de décourrir les syphilitiques éventuclles.

De même, le fait que depuis 1917 nous avons dans le service A de la Maternité, pratiqué là réaction de Wassermamn sur toutes les parturientes admises, a été aussi la cause que plusieurs cas latents 
ont été découverts. En tout cas, il arrive tous les jours que nous trouvions une réaction de Wassermann positive chez des malades qui, cliniquement, ne préscntent aucum signe de syphilis, mai; chez qui un examen plus approfondi confirme que la malade ellemème ou le père de l'enfant a eu autrefois cette affection. Il faut malheureusement constamment compter que bon nombre de ces malades, dans leur ignorance et dans leur légereté, essaient de dissimuler le plus longtemps possible qu'elles ont eu une affection vénérionne.

Nous n'hésitons donc pas à déclarer que la recherche de la réaction de Wassermann chez toutes les parturientes des Maternités des grandes villes nous parait absolument nécessaire.

A en juger daprès nos matériaux, nous croyons done que le chiffre indique originellement par WrLmans, soit environ $5 \%$, est en concordance parfaite avec la réalité et en tout cas, il s'applique à la clientèle qui fréquente les Maternités officielles.

Si on calcule le pourcentage pour les 15,449 accouchements qui ont eu lieu de 1912 à 1921 dans le service A de la Maternité, on constate que ce chiffre est de $5,2 \%$; cependant, comme nous l'avous dejà mentionné, il existe une tendance à l'augmentation dans la dernière année. Il nous semble que ces chiffres indiquent que la syphilis est une affection relativement fréquente chez les parturientes admises dans les grandes Maternités.

Yous n'avons pas lintention de nous occuper en détail de la question de savoir comment la grossesse influence une infection sphilitique existante.

Les matériaux que nous avons à notre disposition ne sont pas non plus aptes à nous renseigner sur la question de savoir jusqu'à fuel point les avortements sont particulièrement fréquents chez les irmmes syphilitiques; cela dépend de ce que les services de Materrité de l'Hôpital n'admettent les parturientes qu'à partir du 5:ème au b:ème mois de la grossesse.

Cependant, d'une manière générale, nous pouvons confirmer les drclarations des auteurs antérieurs (Bettmann, Placzek etc.) :apres lequelles les plaques nuqueuses et d'une manière générale, 1. s manifestations secondaires peuvent atteindre une extension et a.: dimensions qu'on ne rencontre pour ainsi dire jamais en dehors tare grossesse. 
Enfin, nous profiterons de l'occasion pour faire ressortir ce sur quoi KEY et HENDry avaient déjà attiré l'attention, à savoir qu'on observe quelquefois que la réaction de Wassermamn est très variable pendant la grossesse, de sorte qu'une réaction antérieurement très positive peut, sans que la malade ait été soumise à un traitement quelconque, devenir beaucoup plus faible vers le moment de l'accouchement et redevenir fortement positive quelques mois après l'accouchement. Mais cela ne concorde pas avec ce que nous avons observé; il faut se souvenir qu'à l'étranger, en règle générale, on n'examine que la quantité de 0,2 c. c. et cu'une petite différence dans le degré de l'hémolyse peut avoir sa cause dans la technique employée.

Nous allons maintenant parler de ce qui a présenté à notre étude le plus grand intérèt, c. à d. l'influence de la syphilis sur le produit de la gressesse.

Déjà, dans la littérature un peu ancienne, on trouve des statistiques importantes montrant combien est considérable la mortalité infantile quand il s'agit d'une syphilis maternelle non trailée. Nous nous contenterons de citer ici quelques chiffres indiquant la mortalité soit immédiatement après, soit dans le courant des premiers mois; c'est ainsi que Fournier a trouvé une mortalité de $80 \%$, Leduc de $71 \%$, v. Zeiszl, de $80 \%$, Bunchi, de $90 \%$ et Markus de 90,2\%. Ce sont là des chiffres qui démontrent de façon convaincante la gravité de la maladie et, en outre, une autre question se pose, celle de savoir combien parmi les survivants ont, d'une manière générale, été exempts de la maladie.

Si l'on cherche à compléter les chiffres ci-dessus par d'autres moyens, nous trouvons d'abord le premier relevé ancien de FourNIER portant sur 18 familles syphilitiques, dans lesquelles il y eut 151 grossesses dont pas moins de $85 \%$ se terminèrent par la naissance de morts-nés.

Enfin, LePileur a démontré que dans les couples, où la mortalité des enfants était de $3,8 \%$ avant que la mère fut infectée, cette mortalité s'éleva à $79 \%$ après que la mère eut été victime de l'infection.

Dans un travail de Williams et dont les éléments proviennent de Baltimore, cet auteur indique que pour $26,4 \%$ de tous les cas de morts chez les enfants nés après le 7:ème mois de la grossesse et qui moururent dans les 14 premiers jours après l'accouchement, 
c'est la syphilis qui est la coupable. Un autre accoucheur américain, Slemons, confirme les chiffres de Williams en s'appuyant sur d'autres matériaux.

Bishop Herman a comparé les grossesses dans 150 familles syphilitiques et dans 150 familles non syphilitiques et il est arrivé au résultat suivant consigné dans le tableau ci-dessous et calculé pour 1,000 grossesses.

\begin{tabular}{|l|c|c|c|c|c|}
\hline \hline Familles & Grossesses & $\begin{array}{c}\text { Mort-nés et } \\
\text { avorte- } \\
\text { ments }\end{array}$ & $\begin{array}{c}\text { Enfants } \\
\text { morts ulté- } \\
\text { rieurement }\end{array}$ & $\begin{array}{c}\text { Vivants } \\
\text { mais } \\
\text { malades }\end{array}$ & $\begin{array}{c}\text { En bonne } \\
\text { santé }\end{array}$ \\
\hline & & & & & \\
150 sypli. & 1000 & 172 & 229 & 390 & 210 \\
150 non syph. & 1001 & 94 & 114 & - & 792 \\
\hline
\end{tabular}

JeAns a examiné 100 familles syphilitiques dans lesquelles il y eut 331 grossesses.

Dans $30 \%$, les grossesses̉ se terminèrent par avortement; dans $9 \%$ il y ent des mort-nés; dans $61 \%$ les enfants naquirent vivants; mais $24 \%$ moururent ultérieurement.

Ces chiffres tirés de la littérature démontrent suffisamment, nous semble-t-il, quelle est la gravité d'une syphilis non traitée pour le foetus; nous reviendrons plus loin sur les conditions existant pour nos matériaux.

La question consiste maintenant à savoir, jusqu'à quel point, en traitant la mère, on peut obtenir de meilleurs résultats et comment on y arrivera de la meilleure façon.

Il existe sur ce point une incertitude considérable et les diverses cpinions sont en désaccord extrême.

Lne question qui a une grande importance pratique, c'est de savoir ce qui se passe, quand une femme, dont l'infection syphilitique renonte à plusieurs années, vient à être enceinte alors qu'elle a été traitée antérieurement, mais n'a été soumise à aucun traitement specifique au cours de sa grossesse.

Markl's a essayé de donner une réponse à cette question en se fondant sur des éléments, formés de 17 mères, qui, toutes, avaient èté traitées antérieurement. 
Il a trouvé qu'il n'y avait pas moins de $82,3 \%$ d'enfants mis au monde qui présentaient des stigmates de syphilis.

Il est, en outre, intéressant de voir à quelle date remonte l'infection; et on constate que 2 de ces malades avaient acquis leur syphilis 1 à 2 ans avant la conception, 4: 2 à 3 ans avant la conception et 3 avaient été infectées 3 à 4 ans avant la conception; pour 8 malades, le moment de la contamination remontait a 4 ans ou même plus.

Divers auteurs communiquent des résultats analogues et aussi désastreux.

Il ressort également du travail de MARKus qu'on peut obtenir des résultats bien meilleurs en soumettant la mère à un traitement antisyphilitique énergique pendant la grossesse. Sur 46 cas traités pendant la grossesse, $46,5 \%$ seulement des enfants présentèrent des signes de syphilis à la naissance.

Cependant Markus, lui-mème, attire l'attention sur le fait qu'un nombre considérable de ces $46,5 \%$ présenta ensuite des manifestations.

Dans un travail de SAUVAGE, publié en 1913, l'influence du traitement sur les enfants est également étudiée.

Ce travail a une importance d'autant plus grande qu'il tâche d'élucider les résultats du traitement au Salvarsan. Au début, quand on faisait les essais avec le Salvarsan, Ehrlich lui même avait déconseillé cle l'employer chez les femmes enceintes et par la suite un certain nombre d'autres auteurs avaient exprimé la même opinion (Jaeger, Fabre et Bourret, Bar), tandis que d'autres, comme, par exemple, Hüffeli, demeuraient davantage sur l'expectative. On croyait que le Salvarsan ne pouvait être employé dans la grossesse, soit parce qu'il était dangereux pour la mère, soit par qu'il risquait de provoquer un avortement.

Sauvage établit alors que le Salvarsan peut être utilisé dans la grossesse sans trop de risques.

Il n'est pas exact qu'il risque de provoquer l'avortement ou l'accouchement prématuré. SAuvage a été en mesure de suivre 81 cas de femmes enceintes traitées par le Salvarsan. Il est certain que chez 71 d'entre elles, la grossesse s'est terminée par un accouchement quelques semaines avant terme; mais, il s'était écoulé 8 jours à plusieurs mois entre la dernière injection et l'accouchement, de 
sorte que rien ne porte à admettre que le Salvarsan a joué un rôle quelconque sous ce rapport.

Dans 10 cas, l'accouchement se produisit au moment voulu ou presque et dans trois cas seulement la grossesse fut interrompue au 8:ème ou 9:ème mois.

Dans un de ces cas, la malade avait été déjà en traitement pendant deux mois et elle arait reçu en tout 9 injections de Salvarsan et l'accouchement se produisit seulement 8 jours après la dernière injection. Quant aux deux autres cas, il y a également beaucoup de faits qui indiquent que l'interruption prématurée de la grossesse fut due à l'infection syphilitique et non pas au traitement employé.

Sappuyant sur ces résultats et sur des communications de Prxard, Jeanselme, Tourraine et Laignel-Lavastine, Sauvage conclut que, d'une manière généralc, il n'y a pas de grands risques à employer le Salvarsan chez les femmes enceintes. Si l'on voit quelquefois que les douleurs surviemnent à la suite d'une injection, cela se produit prescue toujours dans des cas où la grossesse est presque à terme.

Al contraire, SAUvage est d'avis que le traitement empèche l'accouchement prématuré, qui, ainsi qu'on le sait, est extrêmement fréquent dans la syplilis et il corrobore par des chiffes cette affirmation.

Quant au résultat définitif, le travail de SAuvage comprend 91 cas qu'il a eu occasion de suivre lui-mème de près.

Il nous parait important de mentionner avec quelque détails ces cas qui tous furent traités au Salvarsan pendant la grossesse.

84 des enfants nacpuirent vivants, 2 moururent pendant l'accouchement; il s'agissait de cas avec placenta profondément situé et hémorragie. 5 étaient mort-nés et avaient succombé depuis déjà quelque temps. Quant aux 84 qui naquirent vivants, il en mourut trois qui étaient nés avant terme; deux d'entre eux eurent des hémorragies répétées; 2 nacuirent avec des signes de syphilis congénitale, 2 eurent divers accidents intestinaux et autres maladies infectieuses, mais ne présentaient aucun signe de syphilis; ils furent par la suite observés pendant plusieurs mois; il se développèrent bien et ne présentèrent aucun indice de syphilis.

it étaient en bonne santé apparente au moment de l'accouchement. Cependant cela n'est, évidemment, pas suffisant, car ils 
auraient naturellement pu avoir ultéricurement des manifestations.

Mais Sauvage a observé 23 enfants pendant une période de 2 à 6 mois et sur ces 23 , il y en eut 18 qui continuèrent à bien se porter, 5 eurent des manifestations syphilitiques au cours des 4 premiers mois et 4 d'entre eux succombèrent.

Ainsi qu'on le voit, le nombre de ceux qui ont été suivis pendant assez longtemps est relativement faible; mais les résultats évidents du traitement sont trés marqués. Sauvage se rend bien compte, évidemment, que le fait que les enfants avaient l'air d'ètre en bon état au moment de la naissance ne veut pas dire qu'ils n'auront pas de manifestations ultéricures; cela veut dire uniquement que l'infection syphilitique est peut-ètre latente à ce moment.

Mais, pour avoir des éléments plus considérables et pour pouvoir comparer les différentes méthodes de traitement, Sauvage a donné plus d'extension à ses matériaux, en utilisant des travaux anciens de Pinard, Champetier de Ribes, Boissard et Potocki.

Il a divisé ces matériaux en trois groupes principaux:

Le premier groupe comprend des malades qui ont eu des manifestations au cours de leur grossesse et qui pendant celle-ci ont été traitées seulement au mercure et à l'iodure de potassium.

Le deuxième groupe renferme les femmes qui n'ont pas eu de manifestations pendant la grossesse, qui, avant celle-ci, avaient èté traitées très irrégulièrement, mais qui pendant la période de gravidité ont été traitées régulièrement au mercure et à l'iodure de potassium.

Le troisième groupe, enfin, comprend une série de malades qui ont été régulièrement traitées au mercure et à l'iodure de potassium, tant avant que pendant la grossesse.

Sauvage lui-même tire des conclusions̄ très larges de ces tableaux; mais la seule cloose certaine, ce sont en réalité les chiffres qui indiquent les mort-nés et ceux qui naquirent avec des manifestations syphilitiques.

Le temps d'observation ayant été trop court, on ne peut déterminer combien de syphilis latentes se cachent parmi les sujets sains en apparence. Certainement, le tableau eut été tout autre, si l'on eut examiné les enfants six mois plus tard. 
En résumé, on peut dire que dans le groupe 1 qui contient les malades le plus irrégulièrement traitées il y eut 74,34\% de mort-nés ou morts peu après la naissance; sur ceux qui restent, $10,18 \%$ ont eu la syphilis dans la courte période pendant lacquelle on les a observés. Dans le groupe 2, il y eut seulement $19 \%$ de mort-nés ou d'enfants qui moururent peu après leur naissance.

$14,7 \%$ avaient ou eurent plus tard la syphilis et $66,25 \%$ ne présentaient aucun signe de syphilis pendant la période d'observation.

Le troisième groupe ne nous renseigne à vrai dire que sur un point, en nous montrant que $11,72 \%$ d'enfants étaient des mortnés, tandis que $88,28 \%$ naquirent vivants.

Ce qui est particuliérement marquant dans cette statistique, c'est la chute considérable du pourcentage de mortalité, de $74 \%$ à $11 \%$ et, il nous semble que cela nous permet de conclure avec certitude qu'un traitement au mercure et à l'iodure de potassium pendant la grossesse a une action immédiate considérable.

Mais, il vaut aussi la peine de faire ressortir que si on compare ces séries avec les cas traités au Salvarsan et communiqués par SAuVAGE on trouve un pourcentage de mortalité encore plus bas, soit seulement $7,7 \%$. Mais, ainsi que nous l'avons mentionné plus haut il ne s'agit ici que du résultat momentané et non pas du résultat permanent.

A la suite des publications de Sauvage, divers accoucheurs français vinrent apporter le résultat de leur expérience concernant les effets du Salvarsan.

Lemielland et Brisson communiquent plusieurs cas de la Clinique Tarnier: il s'agit seulement d'un nombre relativement faible de cas et, ce qu'il y a de plus remarquable dans leur travail, c'est leur affirmation qu'ils sont enclins à considérer le Néosalvarsan comme plus dangereux que le Salvarsan pour les femmes enceintes.

Jeanselme, s'appuyant sur 16 cas, essaie de porter un jugement sur le traitement isolé au Salvarsan, qui, dans la majorité des cas, avait été employé à très hautes doses. Sur ces 16 mères, il y en eut deux qui donnèrent naissance à des foetus morts. Ces éléments présentent le même défaut que les précédents, à savoir que les enfants n'ont pas été suivis pendant suffisamment longtemps 
et quand JeAnselme essaie de démontrer, d'après ses cas, que le traitement au Salvarsan est supérieur au traitement mercuriel et ioduré, il ne nous parait pas que ses conclnsions soient suffisamment bien fondées.

La mème objection peut ètre faite à toute une série de travaux du même genre. C'est ainsi que Fabre et Bourrel disent avoir traité 6 femmes enceintes par le Salvarsan et que toutes ces malades ont donné naissance à des enfants sains en apparence. Brisson publie 36 cas, tous traités au Salvarsan, et dans lesquels il y eut 33 naissances d'enfants vivants et sains en apparence et où il n'y avait pas non plus de disproportion entre le poids de l'cnfant et celui du placenta.

v. Szuly mentionne 10 malades ayant toutes une réaction de Wassermann positive et qui, après traitement par le Salvarsan et le Néosalvarsan donnèrent naissance d̀ des cnfants sains en apparence.

Audebert et Berny, d'après leur expérience, recommandent le traitement mixte au mercure et au Salvarsan. En Suède, nous avons une statistique de Hedén: ses résultats avec le Salvarsan seul sont considérablement pires que ceux qu'il a obtenus avec un traitement mixte. aussi bien avec le mercure culavec le Salvarsan.

Enfin, il existe toute une série de communications moins importantes qui ne comprennent qu'un tout petit nombre de cas (Schreiber, Huck, Fräniel et Glaser, y. Zeiszl, Galliot, Levis, Oui, Baisch, Barin, Girauld et Tessier, Eriwin Lange) de sorte qu'on ne peut tirer de conclusions générales de tous ces travaux.

En 1915, parut un travail très considérable d'Erwin Meyer comprenant 43 cas qui avaient été soumis à un traitement mixte pendant la grossesse. Il y eut en tout 42 maissances d'enfants vivants, car une des malades avorta au 6:ème mois. Sur ces 42 enfants, 5 moururent dejà dans les premiers jours qui suivirent la naissance; trois d'entre eux avaient des signes de syphilis. Cependant, dans ces cas, il s'agissait d'enfants dont les mères avaient reçu des doses relativement faibles de Salvarsan pendant la grossesse.

Quant aux 37 autres, 5 d'entre eux avaient déjà au moment de la naissance une réaction de Wassermann positive.

Un certain nombre de ces enfants a été suivi pendant une période assez longue: 3 sont morts dans le premier mois et 2 dans le 
second mois après la naissance; mais, dans la mesure oủ on a pu s'en rendre compte, ils ne présentaient pas de syphilis.

En ce qui regarde les autres enfauts vivants, il n'a pas été possible à Meyer d'examiner personnellement s'ils ont eu des manifestations ultérieurement; mais il est en mesure de dire que deux des enfants vivaient et avaient deux ans, que 19 étaient vivants it l'âge de 1 an et enfin, pour 6 enfants, il n'a été possible de les suivre. que pendant les six premiers mois après la naissance.

Une affirmation de MEYER est également très intéressante: c'est que l'arsenic ne passe pas dans le placenta sain, mais seulement a travers le placenta syphiliticue, opinion qu'il corrobore aussi par des recherches expérimentales.

D'après ce qui précède, on doit considérer comme absolument certain qu'un traitement antisyphilitique pendant la grossesse a une action puissante et que quand, on s'occupe de la question de la syphilis congénitale, il est absolument nécessaire de se rendre compte autant que possible de la façon dont la mère a été traitée pendant sa grossesse.

Cela n'a pas été fait dans un travail de HoRnung paru à une date. aussi récente que 1920. Hoknung a tâché de suivre la voie suivante; il a considéré les résultats en se plaçant au point de vue du stade de la maladie dans lequel se trouvait la mère. Par conséquent, il divise l'infection syphilitique en 4 stades cliniqques, auxquels s'ajoutent en plus la syphilis latente et la syphilis congénitale maternelle. Déjà, cette spécialisation considérable des cas fait courir le danger que les divers groupes seront relativement faibles; mais en outre, le stade de la syphilis auquel se trouve la mère est beaucoup moins important pour l'enfant. Ce qui est capital, c'est de savoir comment le traitement a été pratiqué et quelles sont ses relations avec la grossesse.

Nous ne pouvons non plus accepter, sans plus, l'importance que Hornung attache, dans ses cas de syphilis latente, à l'existence d'une réaction de Wassermann négative ou positive car, cela doit passer au second plan par rapport à l'importance de la question de savoir avec quelle intensité et quelle fréquence les malades ont été traitées.

Quand donc, en terminant, HonNung formule certaines conclusions sans tenir compte de ces points, ses conclusions perdent beau- 
coup de leur valeur et quand il constate les résultats les moins favorables dans les cas où »la syphilis est entrée dans une période de repos" cela peut être plutôt considéré comme une manière de dire que les malades en question ont été insuffisamment traitées.

En revanche, HonNung a raison quand il fait ressortir, en opposition avec Goudy par exemple, que l'influence de l'infection syphilitique sur la grossesse ne sc manifeste qu'après le 4:ème mois. Quand, au contraire, il déclare que $83,3 \%$ des enfants, présentant des signes cliniques de syphillis à la naissance, avaient une réaction de Wassermann négative, ce chiffre est certainement trop élevé; en tout cas, il ne concorde pas avec nos résultats.

L'objection principale à son travail est cependant la suivante: il n'a, pour ainsi dire, pas cherché à trouver une relation entre le résultat final et le traitement auquel ont été soumises les malades en question.

Si l'on veut comparer les résultats des diverses méthodes de traitement, il faut nécessairement des matériaux considèrables. Si par conséquent, Fischl et Steiner trouvent que 6 malades qui n'avaient pas été du tout traitées ou l'avaient été insuffisamment pendant la grossesse donnèrent le jour à des cnfants chez qui la syphilis était peu marquée, alors que 6 autres mères traitées soit avec le Salvarsan, soit avec le mercure dommèrent naissance à des enfants présentant des altérations syphiliticques graves, il parait évident qu'un aussi petit nombre de cas ne permet pas de tirer des conclusions quelconques. Les statistiques de ce genre sont toujours dangereuses à quelque point de vue, quand mème Fiscier et Steiner ne tirent pas de conclusions de leur travail; d'autre part ils ne font pas non plus suffisamment ressortir qu'une telle statistique est sans valeur quand elle ne renferme pas un plus grand nombre de cas.

Beaucoup plus important est un travail de BEck (1921) qui comprend 32 cas de syphilis au cours de la grossesse. Dans 4 cas, le Salvarsan n'a pas été du tout employé pendant la grossesse; dan' 11 cas, on a administré de 1 à 3 injections de Salvarsan $(0,40$ centigr. par dose) et, dans 17 cas, on a administré 5 injections, ou plus encore, de Salvarsan, avant l'accouchement.

En ce qui concerne les 4 premiers cas, tous les enfants naquirent vivants, mais trois d'entre eux présentèrent des signes de sy- 
philis immédiatement ou peu après; ils furent tous traités, mais, d'après BEck, ils ne se développèrent pas aussi bien que les enfants syphilitiques dont les mères avaient reçu du Salvarsan pendant la grossesse.

Quant au quatrième enfant, il fut observé pendant 6 mois saus qu'il eut présenté d'indices de syphilis.

Dans les 11 cas, dont le traitement au Salvarsan avait été relativement peu intense, il y eut un mort-né qui présentait des signes de syphilis; un autre mourut à l'âge de trois mois, également de syphilis et enfin, un enfant, né avant terme, mourut de pncumonie(?) à l'âge de deux mois.

Sur les 8 qui restaient, 3 eurent ou avaient la syphilis, tandis que 5 qui furent observés pendant $1 / 2$ mois, 3 mois, 4 mois $1 / 2,6$ mois et 16 mois ne présentèrent pas de stigmates syphilitiques.

Le demier groupe qui renferme 17 cas est le plus important; c'est, en effet, lui qui renferme les femmes traitées à fond par le Salvarsan au cours de la grossesse.

Deux grossesses se terminèrent par avortement au 5:ème mois; mais les 15 autres enfants naquirent vivants; un avait la syphilis il fut immédiatement traité et se développa bien. Aucun des 14 autres ne présenta un signe quelconque de syphilis; ils furent observés respectivement pendant $1-1-1-2-2$ mois, 4 mois et demi $-6-$ $6-8-10-11-12-15$ et 18 mois.

Bien que les matériaux soient relativement peu abondants, on ne pent pas leur refuser une importance considérable, surtout si on voit combien peu satisfaisants avaient été les résultats antérieurs, ainsi qu'il ressort des chiffres donnés plus haut. Il ne faut pas cependant dissimuler que, immédiatement après la naissance, tous ces cnfants ont reçu un traitement quotidicn avec du mercure pendant près de 2 mois, et il est bien possible que cela a aussi contribué au bon résultat.

Le travail de BEck est américain; mais en Angleterre, aussi, il $y$ a des travaux qui montrent combien le traitement an Salvarsan a de valeur. Il faut en particulier mentionner un travail de FindL.1Y (1921) qui est très précieux par ce que les enfants ont été suivis pendant longtemps; quelques uns d'entre eux jusqu'à l'âge de 7 ans. Il s'agit de 15 cas de traitement au Salvarsan cliez les meres; 14 enfants naquirent vivants, un mourut pendant l'accouche- 
ment. Des examens ultérieurs réitérés ont montré que ces enfants étaient en bonne santé; une seule fois, un a présenté une réaction de Wassermann faiblement positive, mais sans symptômes cliniques.

De plus, Findlay nous apprend que quelques unes des mères en question, sans avoir été soumises à un traitement ultérieur, on donné le jour à des enfants sains; il s'agit en tout de 5 mères et de 10 enfants environ. Le travail de FINDLAY aboutit à réclamer que tous les cas, dans lesquels l'avortement on l'accouchement prématuré sont dus à la syphilis, soient déclarés aux autorités, de façon à ètre sûr que la mère sera soumise au traitement.

ADAns a également suivi une série d'enfants pendant une période relativement longue. Déjà en 1918, il parlait devant la "Royal Society of Medicine» de l'institution de la première clinique vénéréologique pour les femmes enceintes et il donnait des renseignements sur les résultats obtenus et engageait énergiquement à employer le Salvarsan. A la fin de 1920, il déclare que en 1917-1920,95 femnes enceintes ont été traitées au Salvarsan, elles ont donné naissance seulement à 5 mort-nés et que, parnıi les 37 derniẻres qui ont été traitées, ancune n'a donné naissance à des mort-nés. Quatre enfants sont morts par la suite; aucun des enfants nés avec une réaction de Wassermann négative n’a eu ultérieurement une réaction positive ou n'a présenté d'antres signes de syphilis, malgré que beaucoup de ces enfants n'aient pas eu d'autre traitement que celui qu'ils avaient indirectement reç par l'intermédiaire du lait de leur mère.

Il établit donc comme étant son opinion que, si une femme syphilitique enceinte, que sa syphilis soit en activité ou en état de latence, est traitée pendant les 3 à 4 derniers mois avant l'accouchement à l'aide de Salvarsan, il est probable qu'elle dommera naissance a un enfant vivant et sain.

Dans les dernières années, cette opinion que, par un traitement intensif des mères pendant la grossesse, on pourra sauver un grand nombre d'enfants et les mettre à l'abri de la grave affection que représente la syphilis congénitale, cette opinion, disons-nous, est de plus en plus généralement admise.

Les efforts ont donc tendu à constater et à traiter le plus tôt possible dans la grossesse l'infection syphilitique des femmes enceintes. Nous devons mentionner comme une importante étape de 
ces efforts les divers centres érigés dans un certain nombre de villes anglaises et exclusivement consacrés au traitement des femmes syphilitiques enceintes. De même en France, on s'est occupé de cette tâche. Ainsi que nous l'avons dit, à l'instigation de PinARD et de Couvelaire, on a installè à Paris une consultation rattachée à la clinique obstétricale et destinée aux femmes syphiliticues en état de grossesse, et dans lacuelle le traitement est confié à uñ syphiligraphe de profession, ce qui certainement a une importance capitale.

Chez nous aussi, la nouvelle consultation pour femmes enceintes, rattachée aux services obstétricaux du Rigshospital, s'occupe également de cette tâche, car, dès que l'infection syphilitique est constatée, la malade est dirigée sur la clinique vénéréologique de l'Hôpital.

Si on considère nos matériaux du point de vue obstétrical pur, c'est pour se renseigner sur la question de savoir si, pendant l'accouchement ou les suites de couches, il se manifeste des circonstances particulières quand on a affaire à des femmes syphilitiques. La première question intéressante est celle de l'infection puerpérale. En partant de la considération que dans beaucoup de cas, il s'est agi de foetus morts et macérés et de malades qui avaient des manifestations aux. organes génitaux (plaques muqueuses non détergées, suintantes etc.) il n'y avait rien d'invraisemblable à admettre a priori qu'une infection puerpérale se développât chez ces malades, soit pendant l'accouchement, soit plus frécuemment, après.

Au point de vue de la fièvre pendant l'accouchement, il y a eu en tout 12 cas sur 528 dans lesquels les malades sont entrées avec des signes d'infection ou chez lesquelles cette dernière se développa au cours de l'accouchement. Si l'on fait le calcul du pourcentage, on trouve que chez $2,2 \%$ il, y eut un mouvement fébrile pendant l'accouchement et si on désire savoir la répartition dans les différents groupes on obtient le tableau suivant:

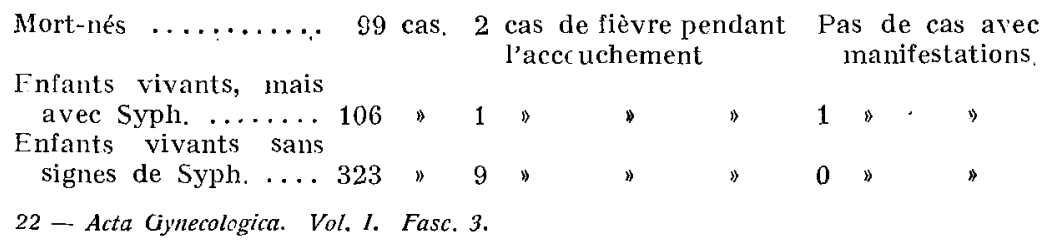


Il ressort déjà de ce tableau que la question de savoir s'il existait ou non des manifestations n'a joué aucun rôle important; et même, on peut aller plus loin et dire que d'une manière générale la syphilis n'a eu aucune influence sur la genèse de l'infection dans les cas que nous mentionnons. Cela devient encore plus évident si on considère les diverses observations. Dans 6 des cas mentionnés, il s'agissait d'élévations tout à fait minimes de température, jusqu'à $38^{\circ} 3$, tout au plus. Le marche ultérieure démontra aussi qu'il s'agissait de cas légers, car deux des malades ne présentèrent pas du tout d'élévation thermique pendant les suites de couches; chez deux il n'y eut de la fièvre que pendant un seul jour, tandis que les deux autres malades eurent de la fièvre durant quelques jours.

Dans quatre cas, il s'agissait d'accouchements prolongés (100117-70 et 48 heures $3 / 4$ ) dans lesquels le liquide amniotique avait été expulsé de bonne heure et où, par suite, les possibilités d'infection de la cavité de l'oeuf avaient existé pendant un temps assez long.

Enfin, nous avons un cas dans lequel la malade entra à la Maternité avec présentation oblique et élévation de température; elle mourut, plus tard, d'infection puerpérale et nous en parlons en détail plus loin: mais c'est là un cas dans lequel il n'existe aucune relation entre la terminaison fatale et l'infection syphilitique.

Il n'y a donc, par conséquent, aucun des cas cités qui nous fournisse quelque motif d'admettre que la syphilis ait joué un rôle tant soit peu important dans la genèse de l'infection. Cela devient aussi nettement manifeste, si on compare ces cas d'élévation de température avec ceux survenus chez les autres parturientes. De 1919 à 1921, sur 9,300 accouchements, nous avons eu en tout 93 cas d'élévation de température au cours de l'accouchement, ce qui donne $2,7 \%$, chiffre qui en tout cas n'est pas inférieur à celui qui a été constaté chez les syphilitiques.

En ce qui concerne la mortalité et la morbidité elles-mêmes, nous trouvons ce qui suit:

Nous avons eu en tout deux cas de mort; dans l'un des cas, il s'agissait d'une malade qui accoucha une heure après avoir été admise à la Maternité, sans avoir été examinée. L'enfant était mort. A la suite de l'accouchement, il survint de l'élévation de température et la parturiente mourut de fièvre puerpérale au bout d'un 
mois. Mais il faut faire remarquer à ce propos que la malade avait eu autrefois la blennorragie et, qu'à l'autopsie, on trouva un abcès ayant son point de départ dans les annexes du côté gauche et il est par conséquent tout à fait logique de mettre en relation sa blennorragie et son infection puerpérale ultérieure.

Dans le second cas déjà mentionné, il s'agissait d'une malade avec présentation oblique.

Elle arriva en état d'infection, mais comme on pensait que l'accouchement était possible par évolution spontanée, on attendit assez longtemps; pourtant il fallut bien finir par intervenir. La malade avait cependant une fièvre élevée et elle mourut 5 jours après l'accouchement. A l'autopsie, on constata de la gangrène de l'utérus et de la péritonite avec salpingite suppurée.

Cette malade avait eu, elle aussi, une blennorragie antérieure.

Il est évidemment difficile de décider si le fait que ces deux malades avaient eu une blennorragie constitue plus qu'un cas fortuit; mais cependant il y a bien des raisons qui indiquent que l'infection fut due à cette affection et non pas à la syphilis.

Si l'on essaie de déterminer la morbidité puerpérale totale on constate que:

La morbidité a été de $29 \%$ pour les malades ayant donné naissance à des enfants morts.

Elle a été de $23 \%$ pour celles qui ont donné naissance à des enfants vivants, avec signes de syphilis.

Elle a été de $17 \%$ pour celles qui ont donné naissance à des enfants vivants sans signes de syphilis.

Si on rassemble tous les cas en un groupe, nous avons 528 cas, ce qui donne une morbidité de $20 \%$.

Comme il n'est pas douteux que toute intervention ou complication augmente la morbidité puerpérale, il sera, également, intéressant de voir comment la morbidité se comporte dans les cas où l'on n'a fait aucune intervention, mais où l'accouchement a été tout à fait spontané et on constate que:

La morbidité pour les naissances de mort-nés a été de . . $22 \%$ Pour les enfants vivants avec signes de Syphilis ..... $18 \%$ Pour les enfants vivants sans signes de syphilis $\ldots \ldots .13 \%$ et que la morbidité totale pour ces. cas a été de ..... $15 \%$ 
Il ressort clairement des chiffres ci-dessus que la morbidité est beaucoup plus considérable dans les cas où il s'est agi de foetus morts ou macérés, que l'accouchement ait été spontané ou qu'on ait été obligé d'employer les ressources de l'art; en effet, la morbidité est de $29 \%$ et de $22 \%$ contre $17 \%$ et $13 \%$ dans les cas où les foetus étaient vivants sans indices de syphilis.

Ces constatations concordent aussi avec un travail de KoTHEN, dans lequel il a recherché la morbidité, non pas seulement dans les cas de syphilis, mais aussi dans les cas, où, d'une manière générale, il s'agissait de parturientes avec foetus morts et, par conséquent, on ne peut pas dire qu'il y ait quelque chose de particulier aux syphilitiques. Ainsi que nous l'avons dit, la morbidité puerpérale totale fut de $20 \%$ pour les parturientes syphilitiques et si l'on compare ce chiffre à la morbidité juerpérale totale pour les années 1912 - 1919, on constate que dans cette série d'années, sur 11,844 parturientes, il y en a eu 2,302 qui ont eu de la fièvre pendant la période puerpérale. 1 Par suite la, morbidité puerpérale devient 19,4, aussi rapprochée du chiffre de $20 \%$ cité plus haut, qu'on puisse raisonnablement le demander.

Il peut, par suite, être important de rechercher, en s'appuyant sur nos matériaux, si cuelcques unes des complications obstétricales les plus ordinaires se rencontrent avec une fréquence particulière chez les syphilitiques. Les complications dont il s'agit ici sont les plus importantes que nous donnons ci dessous:

Dystocie mécanique, positions anormales du foetus, néphrite, éclampsie, placenta praevia et décollement prématuré; pour la période des suites de couches: rétention placentaire (des cotylédons ou des insertions vélamenteuses) ainsi que malformations du foetus et accouchements gémellaires.

Le tableau qui suit (Tableau II) indique les complications les plus ordinaires qu'on ait rencontrées.

On pourrait ètre tenté, dans une certaine mesure de comparer ces chiffres à ceux du service de la Maternité indiquant la fréquence de ces complications chez toutes les parturientes. Mais, comme ces chiffres sont tellement faibles, cela ne nous donnerait qu'une exactitude apparente.

\footnotetext{
1 Nous entendons par là une température rectale de $38^{\circ}$.
} 
TABLEAU II:

\begin{tabular}{|c|c|c|c|c|}
\hline Complication & Mort-nés & $\begin{array}{c}\text { Nés vivants } \\
\text { avec signes de } \\
\text { Syphilis }\end{array}$ & $\begin{array}{c}\text { Nés vivants } \\
\text { sans signes de } \\
\text { Syphilis }\end{array}$ & Total \\
\hline Néphrite & 6 & 6 & 30 & 42 \\
\hline Pyélite ........ & 4 & 2 & 8 & 14 \\
\hline Maladie de coeur & 0 & 0 & 8 & 8 \\
\hline Condylomes...... & 2 & 3 & 10 & 15 \\
\hline Bartholinite & 1 & 1 & 3 & 5 \\
\hline Bassiu rétréci $\ldots \ldots \ldots \ldots$ & 0 & 1 & 0 & 1 \\
\hline Présentation du siêge $\ldots$ & 11 & 1 & 6 & 18 \\
\hline Présentation de la face.. & 1 & 0 & 1 & 2 \\
\hline Présentation obliçue.... & 4 & 0 & 1 & 5 \\
\hline Jumeaux.......... . & 0 & 0 & 3 & 3 \\
\hline Eclampsie $\ldots \ldots \ldots \ldots$ & 0 & 1 & 1 & 2 \\
\hline Placenta prævia........ & 1 & 2 & 0 & 3 \\
\hline Décollement prématuré.. & 1 & 0 & 0 & 1 \\
\hline Rètention -du placenta.. & 2 & 0 & 2 & 4 \\
\hline Rétention des cotylédons & 1 & 0 & 0 & 1 \\
\hline Asphyxie intrautérine .. & 0 & 2 & 11 & 13 \\
\hline Malformations $\ldots \ldots$ & 1 & 0 & 1 & 2 \\
\hline
\end{tabular}

Cependant, on peut conclure de ces chiffres qu'il n'y a pas de complication tellement fréquente qu'il existe un motif quelconque de la mettre en relation avec la syphilis.

Si les présentations du siège, et d'une manière générale, les présentations anormales sont plus fréquentes avec les foetus morts que dans les autres groupes et plus fréquentes que dans les accouchements normaux, cela est dû uniquement au fait que, dans la majorité des cas, il s'est agi de foetus nés plus ou moins avant terme. Et, c'est un fait bien connu que les présentation anormales, telles que celles du-siège, sont d'autant plus fréquentes que la grossesse est interrompue plus précocement.

Il est pourtant important de faire ressortir que la délivrance dans la grande majorité des cas, s'est déroulée sans complications quelconques. Dans 4 cas seulement, la rétention placentaire a donné lieu à des interventions et dans 3 cas, l'expression utérine suivant la méthode de Crédé a suffi pour faire disparaitre la rétention; et dans un seul cas il a été nécessaire de faire le décollement manuel.

En ce qui regarde la rétention des membranes les matériaux de notre travail ne permettent pas d'élucider cette question, car les registres d'observation de la Maternité n'était pas tenus antérieure- 
ment de façon telle qu'on put se rendre facilement compte de cette circonstance. J'ai done borné mes recherches aux années 1919-1920 et 1921 . Nos cas comprennent done 323 syphilitiques et, parmi celles-ci, il y eut 12 fois de la rétention des membranes, c. à d. 3,7.

A titre de comparaison, nous dirons que sur 5,266 accouchements qui ont eu lieu pendant ces mêmes années dans la Maternité, il y eut 223 cas de rétention des membranes, c. à d. 4,2\%. En d'autres termes, l'infection syphilitique ne crée pas une prédisposition spéciale à la rétention des membranes. Cela peut paraitre étonnant en soi, si l'on suppose que l'endométrite syphilitique est une affection fréquente. Cependant cette question est si peu élucidée et tellement discutée que nous ne nous en occuperons pas de façon détaillée ici.

Si les dystocies mécaniques, (qui, certainement dépendent en grande partie des dimensions du bassin et de celles de l'enfant, ainsi qu'on le sait) ont joué un rôle tout à fait secondaire, cela est dû, pour une large part, au fait que, dans beaucoup de cas, les enfants ont été relativement petits. C'est ainsi que le poids moyen des 97 mort-nés fut de 1,887 grammes et celui des 104 enfants vivants, avec signes de syphilis, fut de 2,917 grammes et enfin, de 3,201 grammes, pour les 320 enfants vivants, nés sans aucun signe de syphilis.

Le poids moyen des 521 enfants est 2,899 grammes. Cela montre donc que, en règle générale, les enfants syphilitiques ont un poids très faible, car le poids moyen des enfants arrivés à terme et nés dans une Maternité est indiqué comme étant de 3,333 grammes. Il est difficile de se rendre compte pour quel motif ces enfants ont un poids moindre; cela peut être dû, pour une certaine part, au fait que les enfants sont prématurés; mais cela peut dépendre aussi $\mathrm{du}$ fait qu'ils sont nés à terme, mais sont en état d'hyponutrition.

Comme, justement, le poids est un des critériums, d'après lesquels on décide si un enfant est ou non à terme, nous ne nous risquons pas à tirer des conclusions sûres de nos matériaux qui n'ont pas été étudiés particulièrement en vue de cette question, ni spécialement au point de vue des autres signes de prématurité. Cependant, nous inclinons fortement à admettre que c'est la naissance prématurée qui a joué le rôle principal. A titre de comparaison, 
nous pouvons, en outre, citer le poids moyen de 1,100 enfants, provenant de 1,100 accouchements consécutifs du service $\mathrm{A}$ de la $\mathrm{Ma}$ ternité et pour lesquels, comme pour les cas de syphilis, nous avons seulement éliminé tous les avortements. Le poids moyen de ces 1,100 enfants fut de 3,010 grammes, c'est à dire un peu supérieur à celui de 2,900 grammes trouvé plus haut. Il ressort, de plus, de ces chịfres que, alors que les enfants nés avec des signes manifestes de syphilis pesaient en moyenne 2,900 grammes, les enfants qui, à la naissance, né présentaient aucun indice de syphilis, pesaient au contraire environ 3,200 grammes, c. à d. presque 300 grammes de pliss.

Il pourrait, en outre, être intéressant de savoir si le traitement a quelque influence sur le degré de développement des enfants, surtout sur le poids. Dans ce but, nous avons pour les trois groupes: Mort-nés, enfants avec syphilis manifeste et enfants sains en apparence, cherché à déterminer le poids moyen des enfants traités et des non traités, chacun à part. On constate qu'il n'y a pas grande différence.

Nous avons aussi calculé le poids moyen pour les, enfants des mères traitées au Salvarsan et ce chiffre fut de 3,107 grammes pour 43 enfants.

Quant aux naissances geméllaires, elles n'ont pas été plus fréquentes parmi les syphilitiques que parmi les autres parturientes de l'Etablissement. Une question a joué un grand rôle en obstétrique, c'est celle de savoir s'il n'existe pas quelque relation entre la syphilis et les malformations du foetus. Le travail de Rudaux et de LE LoRier est le plus connu. Ces auteurs pensaient avoir démontré que dans un nombre très considérable de cas de monstruosités. il existait chez la mère une affection syphilitique.

Des auteurs ultérieurs, Holte, Bjørkenheim, Heymann et WEBER n'ont pu cependant confirmer cette assertion et nos cas ne présentent rien qui vienne corroborer cette manière de voir.

Nous n'avons trouvé que deux cas de malformation. Dans l'un, il s'agissait d'un enfant avec malformation des membres supérieurs; il pesait 1,850 grammes, était mort-né et à l'autopsie on ne constata aucun signe de syphilis. Le second cas était un cas de bec-de lièvre. 
Quant aux cas d'avortement chez les femmes syphilitiques, survenus dans l'établissement de 1912 à 1920, leur nombre arrive à 34 seulement ${ }^{1}$.

Comme, pour les motifs que nous avons signalés plus haut, nos matériaux sont relativement peu abondants et ne comprennent que les avortements survenus après le 5:ème mois, nous nous abstiendrons de faire état de nos cas pour tirer des conclusions ayant une grande portée. Il vaut la peine, pourtant, de faire ressortir que 8 seulement de ces malades avaient été traitées pendant la grossesse, tandis que 26 n'avaient pas été traitées pendant leur grossesse et que 9 n'avaient subi aucun traitement."

Dans les pages qui précèdent, nous n'avons même pas mentionné 16 cas dans lesquels les enfants étaient morts pour d'autres causes; c'est pourquoi en terminant nous allons en parler. Dans deux cas, on fit l'accouchement en raison de l'éclampsie, les enfants étant encore viables et dans deux cas, de telles lésions furent produites chez les enfants au cours de la délivrance qu'ils étaient morts au moment de l'accouchement.

Dans 5 cas, les enfants ètaient plus ou moins prématurés et moururent dans les premiers jours; mais dans ces cas, pas plus que dans les autres appartenant à ce groupe, on ne constata à l'autopsie de signes quelconques de syphilis.

Dans un cas, il s'agissait de pneumonie grippale chez la mère; dans un cas de bassin rétréci, on fit une basiotripsie; dans un autre cas, la morst fut due à un décollement prématuré du placenta; dans un autre, l'enfant naquit en prèsentation de la face et mourut ultérieurement d'hémorragie intracranienne; dans un cas, sans cause connue, il se produisit de l'asphyxie intra-utérine; dans un autre cas, un enfant mourut le 4:ème jour sans cause démontrable et enfin, dans un cas, il s'agissait probablement d'infanticide; l'enfant fut apporté mort, après être né au domicile de la parturiente, dans des circonstances suspectes.

Dans tous les cas que nous venons de mentionner, l'autopsie a été pratiquée et chez aucun de ces enfants on n'a trouvé d'indice de syphilis. Il est très possible que le fait de faire entrer quel-

${ }^{1} \mathrm{Si}$ dans les pages qui suivent, nous mentionnons un nombre plus considérable d'avortements, ces chifíres proviennent de la clientèle particulière et de celle de la Policlinique. 
ques uns de ces enfants dans ce groupe soit discutable et il est très possible que, parmi eux, il se soit trouvé des enfants qui eussent présenté plus tard des signes de syphilis, s'ils avaient vécu. Mais, nous avons jugé qu'il était plus prudent de placer ces cas dans un groupe séparé.

Dans les pages qui précèdent, nous nous sommes occupés, d'une part, des travaux antérieurs sur le traitement de la syphilis pendant la grossesse, d'autre part nous avons montré que la syphilis n'est pas une complication jouant un rôle important dans l'accouchement ou dans les suites de couches; nous allons maintenant tracer les lignes capitales des faits exposés dans le deuxième chapitre principal de notre travail; dans ce chapitre, sont cantenus nos matériaux personnels et les résultats que nous croyons pouvoir en déduire.

Il s'agit d'un côté, au moyen de nos cas, de corroborer et de confirmer les recherches antérieures et d'un autre côté de les compléter et éventuellement de les rectifier.

Nous voulons essayer de démontrer:

Quels sont les résultats pour les enfants, quand la syphilis maternelle n'est pas traitée.

Quels sont les résultats, quand la mère a été antérieurement traitée, mais ne l'est pas au cours de la grossesse; ici cependant il faut faire entrer en ligne de compte aussi bien le traitement qui a été employé, que la période qui s'est écoulée entre le dernier traitement et la grossesse actuelle.

Il est très important de rechercher quels sont les résultats quand la mère est aussi traitée pendant la grossesse, et de rechercher également si le traitement au Salvarsan est supérieur aux autres; il ne s'agit pas du résultat momentané, mais surtout du résultat définitif. C'est pour cette raison que pour porter un jugement sur ce point-là, on ne peut utiliser que les cas dans lesquels les enfants ont été observés pendant une période suffisamment longue.

Pour terminer, nous essaierons, èn nous fondant sur notre expérience, de donner des règles générales sur le traitement des femmes syphilitiques pendant la grossesse et de rechercher, dans quelle mesure les exigences que nous croirons devoir formuler ont été généralement accueillies dans notre pays.

Pour résoudre les problèmes posés ci-dessus, nous avons essayé de suivre le plus grand nombre possible d'enfants nés à la Mater- 
nité, de mères syphilitiques, de manière à pouvoir ainsi nous rendre compte a quel degré la différence de traitement ou l'absence de traitement influe sur la descendance. Il va sans dire que c'est là une tâche qui a présenté pas mal de difficultès. Le premier obstacle et le plus important; c'est évidemment celui résultant du fait que les malades de la Maternité sont traitées secrétement, c'est à dire qu'elles sont à la Maternité sans donner leur nom. C'est là un obstacle qui, à première vue, pourrait paraitre insurmontable, mais, heureusement, il n'en a pas été ainsi. En effet sur toutes les observations de la Maternité, sont consignés le jour et l'année de la naissance de la mère. Si les mères ou les enfants présentent des manifestations syphilitiques, on les transfère le plus ordinairement dans le service de vénéréologie, ce que l'on consigne sur l'observation. Par exemple: "Transférée à l'Hôpital Rudolph Bergh avec son enfant le 1:er mai 1917." Si maintenant, on parcourt le registre d'entrées de ce service au jour dësigné, on y retrouve une ou plusieurs des malades de la Maternité avec leur nom transférées avec leur enfant pour syphilis. D'après le sexe de l'enfant et l'âge de la mère, on pourra alors, dans la majorité des cas, identifier la mère. Au moyen d'un fichier qui se trouve maintenant dans la plupart des services de vénéréologie, on pourra arriver à une identification absolument certaine de la mère et trouver son observation dans le service. En règle générale, on pourra retrouver aussi l'observation de l'enfant; cependant cela présente parfois un peu plus de difficultés. En effet, l'enfant est ordinairement inscrit avec la dénomination »garçon ou fille non baptisé» (c. à d. non dénommé); pendant le séjour dans le service hospitalier, il arrive assez fréquemment que l'enfant reçoive le nom du père et non pas celui de la mère. Comme ce n'est pas le nom du père, mais celui de la mère qui importe, on n'a pas d'autre ressource que de feuilleter tous les régistres d'observations postérieurs à la date d'entrée et de rechercher un enfant qui aura été en observation ou en traitement pour syphilis congénitale. De cette manière, nous avons pu dans tous les cas, réussir à trouver tel ou tel enfant. Il ressort de l'observation de notre service, soit que l'enfant avait ou a eu la syphilis congénitale et alors la chose va de soi, ou bien l'enfant a été en observation pendant 15 jours ou un mois sans avoir présenté de symptômes, ce qui évidemment ne veut rien dire du tout; mais, nous avons l'adresse de l'enfant et nous pouvons le 
faire venir. Les conditions sont beaucoup plus compliquées quand les malades en sortant de la Maternité ne sont pas allées dans un service hospitalier, mais sont rentrées chez elles (»rentrée chez elle avec son enfant"). Cependant, sur l'initiative due à Leopold Meyer - il est toujours indiqué dans l'observation que les malades ont été traitées en telle ou telle année, dans tel ou tel service de vénéréologie, par tel ou tel nombre de traitements ou d'injections de Salvarsan. En feuilletant alors les registres de l'année correspondante dans ce service spécial, on réussit, dans la majorité des cas, à retrouver la malade cherchée. En règle générale, d'après l'âge et la nature du traitement, on peut l'identifier d'une manière absolument certaine et on a ainsi son nom et son adresse. De cette façon, en nous fondant sur l'adresse, nous avons convoqué des malades et leurs enfants. Malheureusement, nous avons constaté qu'un certain nombre de ces malades avait déménagé entre temps; un certain nombre ne se rendit pas à notre appel, bien que l'adresse fut exacte. Il fallut donc écrire de nouveau, parfois à plusieurs reprises et, éventuellement, aller voir ces malades. Nous avons réussi de cette façon à retrouver en tout $68 \%$ des malades de la Maternité et leurs enfants. Il est évident que cela fut une tâche formidable; il nous a fallu plusieurs années pour rechercher et examiner toutes ces malades et leurs enfants; mais nous avons réussi ainsi à réunir des matériaux beaucoup plus considérables que ceux qui ont été examinés dans n'importe quelle autre partie du monde. De plus, aux malades de la Maternité, nous avons ajouté environ 100 cas de notre clientèle particulière ou de la policlinique. Les résultats ressortent du tableau ci dessous. Nous voulons seulement faire immédiatement remarquer que les enfants qui sont indiqués comme sains ont été suivis tous, cliniquement et sérologiquement, pendant au moins 6 mois après leur naissance, la majorité pendant plus longtemps et même 15 ans après leur naissance. On affirme généralement que, un enfant syphilitique présente des manifestations 3 mois au plus tard après sa naissance, de sorte qu'une période d'observation de 4 mois serait suffisante pour décider si un enfant issu de parents syphilitiques est sain ou non. Cependant, cette affirmation n'est pas exacte, car, dans quelques rares cas, les manifestations peuvent survenir plus tard. C'est ainsi, qu'une seule fois, nous avons vu un enfant qui eut des manifestations 5 mois après la naissance. Les enfants 
que nous n'avons pu retrouver se répartissent d'une manière égale dans tous les groupes du tableau ci-dessous, de sorte qu'il n'existe pas d'erreur du fait de l'absence d'un nombre particulièrement considérable d'enfants dans un des groupes.

TABLEAU 1II. TABLEAU SYNOPTIQUE,

\begin{tabular}{|c|c|c|c|}
\hline $\begin{array}{l}\text { Trailement de la maladie } \\
\text { chez la mère. }\end{array}$ & $\begin{array}{c}\text { Nombre } \\
\text { de cas }\end{array}$ & $\begin{array}{l}\text { Enfants } \\
\text { avec } \\
\text { syphilis }\end{array}$ & $\begin{array}{c}\text { Enfants } \\
\text { sains }\end{array}$ \\
\hline 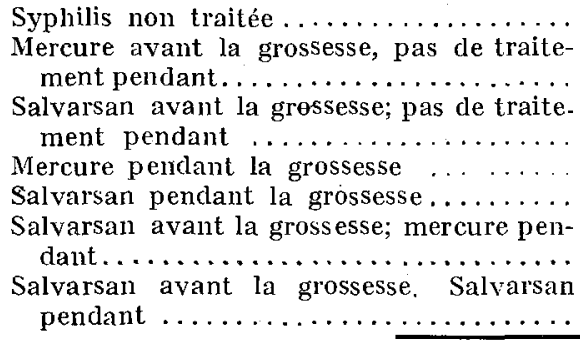 & $\begin{array}{r}158 \\
87 \\
15 \\
111 \\
79\end{array}$ & $\begin{array}{r}157 \\
78 \\
12 \\
80 \\
19 \\
7\end{array}$ & $\begin{array}{r}1 \\
9 \\
\\
3 \\
31 \\
60 \\
19\end{array}$ \\
\hline Tot & 483 & & \\
\hline
\end{tabular}

Pour faciliter la vue d'ensemble, trois mères qui ont donné le jour à des jumeaux sont indiquées comme formant 6 cas. L'une des mères de ces jumeaux se trouve parmi les femmes non traitées; les deux autres sont dans le groupe 2 (mercure avant la grossesse; pas de traitement pendant la grossesse); les 6 enfants eurent tous la syphilis.

1) Syphilis non traitée.

Si nous considérons d'abord la rubrique "Syphilis non traitée" nous voyons que nous avons examiné 158 mères syphilitiques, qui n'avaient jamais reçu le moindre traitement spécifique. Sur ces enfants, 157 ont eu la syphilis, un seul est demeuré sain, résultat réellement triste. Si nos recherches démontrent un nombre beaucoup plus considérable d'enfants syphilitiques que tous les travaux publiés antérieurement, la raison est simplement la suivante: c'est que dans tous les cas, nous avons suivi les enfants pendant au moins 6 mois après la naissance. Dans pour ainsi dire tous les articles publiés avant nous, il figure toujours un nombre considérable d'enfants vivants, sans syphilis et sans réaction de Wassermann; 
mais dans la majorité des cas, les enfants n'ont été suivis que pendant un mois tout au plus, après leur naissance.

Cependant nous devons faire une réserve. En plus des 158 enfants mentionnés, il y en eut 41 que nous n'avons pu retrouver. Il est possible que parmi eux il s'en soit trouvé quelques uns au moins qui sont demeurés sains. Cette hypothèse acquiert de la vraisemblance par le fait, qu'il est naturellement toujours plus facile de retrouver les enfants qui ont eu la syphilis que ceux qui ont continué à bien se porter. Si, cependant, nous nous en tenons à ce que nous savons réellement, nous obtenons le résultat mentionné qui est très déplorable.

Sur les 157 mères d'enfants syphilitiques, 78 avaient des manifestations secondaires, soit une première manifestation, soit une récidive d'une manifestation non traitée auparavant; 79 avaient une syphilis latente avec réaction de Wassermann positive. De ces dernières, 4 avaient de la leucodermie; il s'agissait donc chez elles d'une syphilis qui, probablement, avait été contractée dans les derniers trois ans. Dans un cas, l'infection remontait, à coup sûr, à 5 ans en arrière, car la malade avait avorté 4 fois avec un an d'intervalle chaque fois. Dans tous les autres cas de syphilis latente, il était impossible de determiner l'âge de l'infection, car les malades ne soupçomaient pas leur affection et parce qu'il n'existait pas de signes objectifs. Chez deux malades avec manifestations secondaires, il s'agissait de syphilis postérieure à la conception; les malades avaient été infectées respectivement au 5:ème et 6:ème mois de la grossesse et toutes deux accouchèrent d'enfants syphilitiques. Pour les autres, nous ignorons à cuel moment l'infection s'est produite, soit avant soit après la conception, soit au même moment.

Dans les cas où on a fait le titrage de la réaction de Wassermann chez les mères qui, toutes sans exception, donnèrent une réaction positive, le résultat fut le suivant.

TABLEAU IV.

Dose minimum en c. c. Nombre des mères avec produisant l'inhibition syphilis secondaire

$0,2 \quad \ldots \ldots \ldots 6$

$0,1 \quad \ldots \ldots \ldots \ldots$

$0,05 \quad \ldots \ldots \ldots \ldots$
Nombre des mères avec syphilis latente 
Dose minimum en c. c. Nombre des mères arec Nombre des mères avec prod:isa .t l'inhibition syphilis secondaire syphilis latente

$\begin{array}{llrr}0,025 & \ldots \ldots \ldots & 15 & 8 \\ 0,01 & \ldots \ldots \ldots & 5 & 1 \\ 0,005 & \ldots \ldots \ldots & 0 & 1 \\ 0,0025 & \ldots \ldots \ldots & 2 & 0 \\ 0,011 & \ldots \ldots \ldots & 1 & 0\end{array}$

Ainsi qu'on le voit, on trouve un nombre relativement considérable de réactions faibles dans la syphilis latente. Le tableau confirme tout à fait ce que BoAs avait trouvé antérieurement, à savoir que la syphilis secondaire réagit en moyenne plus fortement que la syphilis latente.

Sur les 157 enfants atteints de syphilis, il y eut 16 avortements après le 6:ème mois, 4 prématurés, 67 étaient mort-nés avec syphilis et plus ou moins macérés; 70 naquirent vivants. Sur ce dernier nombre, 16 avaient des manifestations à la naissance, 28 paraissaient sains, mais avaient à la naissance une réaction de Wassermann positive; de ces derniers, 13 eurent, peu après, dans manifestations syphilitiques. Le reste fut traité dans les divers services de vénéréologie par le traitement spécifique. Les 26 autres étaient en parfaite santé au moment de la naissance avec Wassermann négatif; mais tous eurent plus tard des manifestations syphilitiques. Chez 8 , la réaction positive de Wassermann se manifesta une ou deux semaines après la naissance et la syphilis apparut peu après. Pour le reste des cas, la syphilis congénitale fut constatée respectivement 1 mois ( 8 cas), 2 mois ( 3 cas) 3 mois ( 4 cas) et 4 mois ( 1 cas) et enfin dans un cas la syphilis congénitale fut découverte chez un enfant seulement deux ans après la naissance; mais, evidemment, elle avait existé depuis longtemps déjà. La mère était une jeune fille avec syphilis latente (réaction de Wassermann 20,100-10,100 et 20,100). Au moment de la naissance, l'enfant avait un Wassermann négatif et pas de signes de syphilis. Dans le service N:o IV de l'hôpital municipal on. lui donna préventivement du calomel (mais seulement 19 centigrammes). On enjoignit à la mère de se représenter pour un contrôle prolongé, mais elle ne revint pas. Deux ans après, ayant retrouvé l'enfant nous le trouvâmes bien développé; il ne présentait aucun signe clinique de syphilis, mais, à deux reprises, il eut une réaction de Wassermann très positive $(0 ; 0,060,100)$. 
Ainsi que nous l'avons dit, un seul enfant demeura sain. La mère ètait une jeune fille de 16 ans avec des manifestations généralisées de syphilis secondaire dont elle ne soupçonnait pas l'existence. L'enfant naquit à terme et, à la naissance, il avait une réaction de Wassermann négative. La mère et l'enfant furent transférés à l'Hôpital Rudolph Bergh où l'enfant demeura en observation pendant un délai d'un mois après la naissance; à plusieurs reprises, on constata qu'il avait une réaction de Wassermann négative, avec absence constante de symptômes cliniques. Sept ans après la naissance, nous réussimes à retrouver cet enfant; c'était une fillette développée d'une façon absolument normale, sans les moindres symptômes cliniques et avec une réaction de Wassermann négative. Pour plus de certitude, nous examinâmes cette enfant un an après; elle avait toujours un Wassermann négatif. Il est hors de doute que dans ce cas cette enfant a échappé à l'infection. Il est difficile de dire pourquoi cette enfant occupe ainsi une place à part. On peut s'imaginer deux possibilités: la mère aurait été infectée très tardivement dans sa grossesse; mais à cela on peut objecter qu'elle avait déjà des manifestations secondaires et l'on connait bon nombre de cas de syphilis congénitale, dans lesquels la mère avait seulement le chancre primitif. Ou bien encore, on peut supposer que la mère a peut-être su qu'elle avait la syphilis et qu'elle s'est traitée sans en rien dire.

La mortalité a été très considérable parmi les enfants nés vivants. Nous ne pouvons cependant donner des chiffes déterminés parce qu'il ne nous a pas été possible de suivre tous les enfants syphilitiques. Le pronostic, en dehors de la gravité de l'infection, dépend évidemment, en première ligne, du traitement plus ou moins énergique (par exemple du fait que les enfants, en plus du mercure, ont aussi reçu du Salvarsan), de la nutrition (si les enfants ont été nourris au sein ou au biberon), des soins qu'on leur donue, de l'hygiène générale etc. Un nombre assez considérable d'enfants qui, à la naissance, étaient en bonne santé apparente; et avaient une réaction de Wassermann négative, a succombé par la suite aux manifestations. Du reste, la question de la mortalité dans la syphilis congénitale est suffisamment élucidée dans notre littérature nationale; nous rappellerons seulement les grandes statistiques de Ehlers et les statistiques moins étendues de Lomhol et de Boas. 
Chez les enfants dont la réaction de Wassermann fut titrée à la naissance, l'intensité de la réaction fut la suivante:

TABLEAU V.

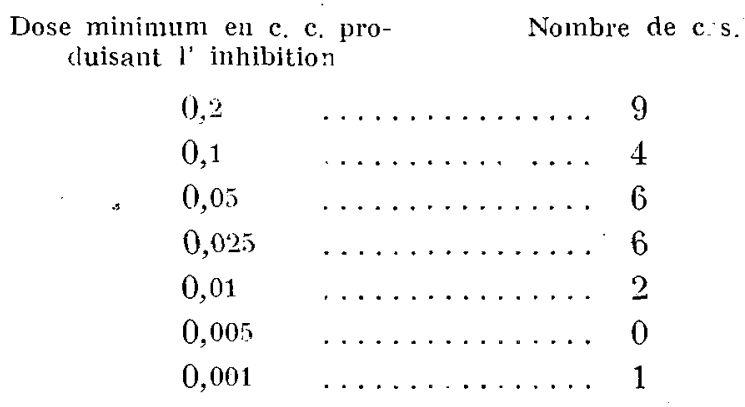

Le résultat principal de nos recherches sur les mères avec syphilis non traitée est donc que celles-ci donnent presque toujours naissance $\grave{a}$ des enfants syphilitiques.

2.) Traitement mercuriel avant la grossesse. Pas de trailement au cours de celle-ci.

Nous avons examiné 87 mères; 78 enfants issus de ces mères avaient eu la syphilis; 19 seulement étaient sains. Le traitement mercuriel administré varie du traitement simple avec 30 frictions jusqu'au traitement intermittent suivi très consciencieusement pendant 3 ans. Le laps de temps écoulé entre le dernier traitement et le début de la grossesse varie entre quelques mois et 20 ans. Si l'on sépare les cas qui ont suivi un traitement mercuriel relativement sérieux pendant deux ans au moins, mais ne l'ont pas suivi pendant la grossesse, on trouve pour ces cas: 7 mères qui ont donné naissance à 5 enfants syphilitiques et à 2 enfants sains, c. à d. donc, qu'il n'y a pas une différence particulièrement remarquable avec les autres cas au nombre de 80 , qui ont eu 73 enfants syphilitiques et 7 enfants sains. De même, le laps de temps écoulé entre la cessation du traitement et l'apparition de la grossesse ne joue pas un rôle aussi considérable qu'on l'admet généralement; c'est là, du reste,un point très important, sur lequel nous reviendrons plus loin. Sur 50 enfants, nés entre 3 et 20 ans après l'infection, 44 étaient syphilitiques et 6 étaient sains, tandis que parmi les autres nés dans les 
trois premières années après l'infection, il y eut 34 syphilitiques et 3 en bonne santé, différence qui n'est pas particuliérement imposante. Comme argument très sérieux, nous devons citer que 4 enfants nés après la 16:ème année qui suivit l'infection furent tous syphilitiques. Dans aucun des cas, autant qu'on en peut juger par les observations, il n'existait de syphilis postconceptionnelle. Deux seulement des mères avaient eu des manifestations, l'une avait une manifestation secondaire généralisée, la syphilis datant de 3 ans; elle donna naissance à un mort-né avec des lésions syphilitiques dans les organes; l'autre åvait une manifestation tertiaire (gommes cutanées et périostiques); la syphilis datant chez elle de 8 ans. Elle donna naissance à un enfant vivant atteint de syphilis.

Chez les autres mères qui donnèrent le jour à des enfants syphilitiques, la réaction de Wassermann a été faite 67 fois. Chez 6 elle a été négative à la suite du traitement antérieurement fait; chez les autres, elle a été positive. L'intensité ressort du tableau ci dessous; le titrage n'a été fait que chez 36 seulement.

TABLEAU VI.

$\begin{gathered}\text { Dose inhibitrice } \\ \text { minimum en c. c. }\end{gathered}$
0,2
min

7 mères qui avaient accouché d'enfants sains, avaient toutes une réaction de Wassermann négative.

Parmi les enfants des syphilitiques, il y eut 16 avortements, 6 naissances prématurées, 18 mort-nés avec syphilis et 38 enfants vivants, dont 29 eurent des manifestations de syphilis à la naissance, tandis que 9 étaient sains en apparence. Cependant tous ces derniers eurent des manifestations de syphilis congénitale, respectivement une semaine (1), 2 semaines (2) 1 mois- (3) 2 mois (1) et 3 mois (2) après la naissance. Chez 4 , la réaction de Wassermann positive apparut 1 semaine avant la manifestation, chez le reste, elle apparut en même temps que cette manifestation.

23 - Acta Gynecologia. Vol. I. Fasc. 3. 
Les 9 enfants sains ont été observés pendant un laps de temps allant de 6 mois à 15 ans après la naissance et constamment on les a trouvès sans symptômes cliniques ni sérologiques.

Chez les enfants qui eurent une réaction de Wassermann positive, celle-ci eut l'intensité suivante:

TABLEAU VII.

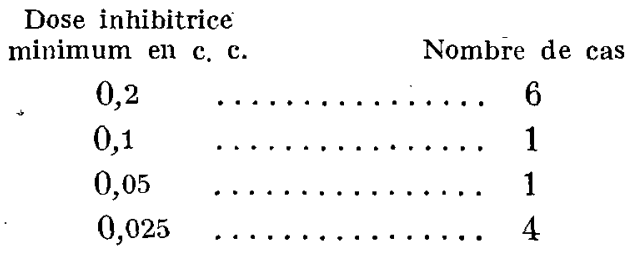

3) Traitement au Salvarsan avant la grossesse; pas de traitement au cours de celle-ci.

Nous avons examiné 15 mères; 12 eurent des enfants syphilitiques et 3 seulement, des enfants sains, résultat qu'on ne peut considérer en aucune façon comme particulièrement remarquable, bien que il ait été un peu meilleur que chez les malades qui avaient été traitées auparavant par le mercure. La plupart des mères dont nous parlons ici avaient reçu un simple traitement mixte au mercure et au Salvarsan au début de leur affection: dans la majorité des cas, une injection intra-musculaire de Salvarsan à 60 centigr. et une injection intraveineuse de 40 centigrammes, associées avec 50 frictions à 3 grammes. Deux malades, à quatre mois d'intervalle, avaient reçu exactement le mème traitement en raison de manifestations. Deux autres avaient reçu 3 injections de Salvarsan avec en plus 50 frictions. La différence de traitement ne s'est pas traduite dans les résultats. Les 2 malades qui avaient reçu à deux reprises du mercure et du Salvarsan eurent toutes deux des enfants qui moururent de syphilis. La seule malade qui, outre le traitement au mercure et au Salvarsan du début, avait subi aussi à titre préventif 300 frictions dans les trois premières années de sa maladie, eut, 4 ans après l'infection, un foetus macéré avec altérations syphilitiques et réaction de Wassermann très positive. Toutes les malades avaient été soumises au traitement quand la manifestation secondaire avait fait son apparition complète et dans aucun des cas, il n'y avait de syphilis post-conceptionnelle. L'âge de l'infection lors de la grossesse ne révéla aucune différence ainsi que le montre le tableau suivant. 
TABLEAU VIII.

Nombre d'années après le premier traitement au Salyarsan
Enfants syphilitiques
Enfants sains

0

1

0

0

1

Ainsi que Thomsen et Boas l'ont déjà démontré, la réaction de Wassermann chez la mère au, moment de l'accouchement a une certaine importance pour le pronostic. Chez 8 des 12 mères d'enfants syphilitiques, nous pratiquâmes la réaction de Wassermann; chez 7, elle fut positive et chez une seulement, elle fut négative; néanmoins, l'enfant eut des manifestations syphilitiques 3 semaines plus tard. Les trois mères d'enfants sains avaient toute une réaction de Wassermann négative. L'intensité des réactions positives ressort du tableau ci-dessous:

\section{TABLEAU IX.}

Dose inhibitrice minimum en c. c. Nombre de cas

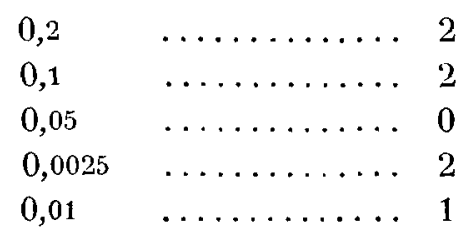

En ce qui concerne la descendance des femmes syphilitiques on trouva 2 avortements après le 6 :ème mois, 5 mort-nés et 5 enfants vivants avec syphilis. Sur ces 5 enfants vivants, 3 avaient des manifestations à la naissance, 1 avait une réaction de Wassermann positive et 1 semblait en bonne santé à la naissance, mais trois semaines après, il présenta des manifestations et succomba à l'âge de 8 semaines. L'intensité de la réaction de Wassermann fut titrée seulement dans 3 cas; il s'agit de réactions relativement faibles. 
Dose inhibitrice minimum en c. c. Nombre de cas

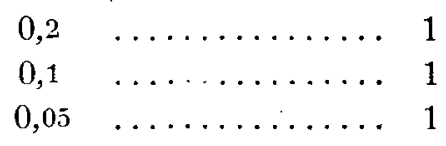

Les trois enfants nés en bonne santé ont été observés 1 an, 3 ans et 6 ans après la naissance et on peut donc admettre probablement à coup sûr qưils ont échappé à l'infection.

4) Traitement mercuriel pendant la grossesse avec ou sans trailement mercuriel antërieur.

Nous avons examiné 111 mères: 80 enfants ont eu la syphilis; 31 seulement demeurèrent en bonne santé, c. à d. donc moins d'un tiers, résultat qui n'est brillant à aucun point de vue et qui surtout est beaucoup inférieur à ce que nous attendions. Nous avons essayé de diviser nos cas de façon à avoir d'un côté les mères qui ont reçu du mercure seulement pendant la grossesse et pas de traitement antérieur, et d'un autre côté celles qui avaient reçu du mercure avant et pendant leur grossesse. Le nombre de ces dernières est de 37, dont 25 eurent des enfants syphilitiques et 12 seulement des enfants sains c. à d. une proportion tout à fait semblable à ce que l'on trouve si l'on regarde le tout en bloc. Pour ne pas obtenir des nombres trop faibles, nous avons renoncé à cette division.

Nous devons faire, d'emblée, remarquer que pour pouvoir parler de traitement pendant la grossesse, nous avons exigé un traitement fait au moins pendant un mois. Les cas dans lesquels la mère commença un traitement 15 jours avant l'accouchement ne sont évidemment pas compris là dedans. Presque toujours, le traitement employé a consisté en frictions; un très petit nombre de parturientes seulement a reçu des injections mercurielles. L'intensité du traitement a varié, depuis une seule cure de 30 frictions jusqu'à un traitement soigneusement éxécuté, avec cure préventive pendant la grossesse. Si on rassemble les cas qui ont subi un traitement véritablement fait à fond (au moins trois fois) pendant la grossesse, le résultat devient heureusement bien meilleur. 37 mères qui avaient reçu un traitement de ce genre ont eu seulement 17 enfants syphilitiques, tandis que 20 demeurèrent sains; différence singulièrement 
frappante avec le nombre total de celles traitées au mercure. Parmi ces malades, on n'en trouve aucune de la clientèle particulière, car, pendant le temps que nous avons exercé, nous avons toujours employé le Salvarsan. Nous ne doutons pas que les résultats ne fussent bien meilleurs encore, si on pouvait présenter une importante statistique tirée de la clientèle particulière. Il faut, en effet; se souvenir qu'il existe une différence considérable entre les jeunes femmes ayant une situation de fortune leur permettant de faire tout ce qu'elles peuvent pour avoir des enfants sains et les malheureuses servantes et ouvrières d'usine pour lesquelles l'enfant attendu (en tout cas au moins très souvent) ne représente qu'un surcroit nouveau dans leur misère sociale; ces femmes ne se donnent pas la peine de faire un traitement tant soit peu soigneux dans l'intérêt de cet enfant. Comme il fallait s'y attendre, le fait que les malades avaient ou non des manifestations a joué un rôle considérable, ainsi que le montre le tableau auivant:

TABLEAU XI.

\begin{tabular}{|c|c|c|c|}
\hline $\begin{array}{l}\text { Nature de la maladie } \\
\text { pendant la grossesse. }\end{array}$ & $\begin{array}{l}\text { Nombre } \\
\text { de cas }\end{array}$ & $\underset{\text { syphilitiques }}{\text { Enfants }}$ & $\begin{array}{c}\text { Enfants } \\
\text { sains }\end{array}$ \\
\hline Syph. Secondaire & 37 & 33 & 4 \\
\hline Syph. Tertiaire . & 1 & 0 & 1 \\
\hline Syph. Latente & 73 & 37 & 26 \\
\hline
\end{tabular}

Ainsi qu'on le voit, le nombre relativement le plus grand d'enfants syphilitiques fut engendré par les mères ayant des manifestations secondaires au cours de la grossesse.

On n'a pas constaté que l'ancienneté de l'affection ait eu une signification pronostique importante pour la descendance. 9 mères dont la grossesse se produisit entre 4 et 11 ans après l'infection donnèrent naissance à 7 enfants syphiliticues et seulement à 2 enfants sains; les deux enfants sains furent engendrés 5 et 8 ans après l'infection maternelle; les 7 syphilitiques naquirent 4 ans (5), 5 ans (1) et 11 ans (1) après que la mère eut été contaminée.

Chez 41 malades, mères d'enfants syphilitiques, nous avons fait la réaction de Wassermann lors de l'accouchement; 12 eurent une réaction négative, et 29 une réaction positive. L'intensité ressort du tableau ci-dessous: 
TABLEAU XII.

Dose inhibitrice minimum en c.c.

Nombre de cas

$\begin{array}{llr}0,2 & \ldots \ldots \ldots \ldots \ldots & 6 \\ 0,1 & \ldots \ldots \ldots \ldots \ldots & 6 \\ 0,05 & \ldots \ldots \ldots \ldots \ldots & 11 \\ 0,025 & \ldots \ldots \ldots \ldots \ldots & 4 \\ 0,01 & \ldots \ldots \ldots \ldots & 0 \\ 0,005 & \ldots \ldots \ldots \ldots & 2\end{array}$

Chez 15 malades, mères d'enfants sains, nous pratiquâmes la réaction de Wassermann; chez 12 , elle fut négative, positive chez 3 ( 2 fois avec 0,2 c. c. 1 fois avec 0,025 ).

Ici aussi, on constate que la réaction de Wassermann chez la mère a une certaine importance pronostique pour l'enfant, bien que la différence ne soit pas aussi frappante, quand la mère a été en traitement pendant la grossesse que lorsqu'elle n'a pas été traitée.

Parmi la descendance syphilitique, il y eut: 5 avortements après le 6:ème mois, 2 prématurés, 36 mort-nés et 37 enfants vivants. Sur les 37 vivants, 31 avaient des manifestations ou une réaction de Wassermann positive au moment de la naissance; 6 paraissaient sains à la naissance avec réaction de Wassermann négative; mais 5 mois au plus, après leur naissance, ils eurent une réaction de Wassermann positive et des manifestations syphilitiques. L'intensité de la réaction de Wassermann a été titrée chez 8 .

TABLEAU XIII.

Dose inhibitrice minimum en c. c.

Nombre de cas

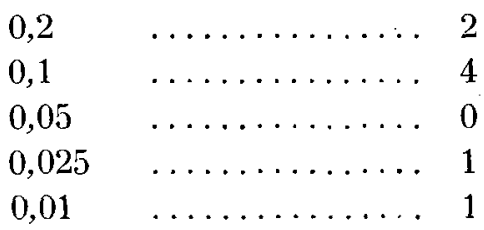

Les 31 enfants en bonne santé ont été observés pendant une période variant de 6 mois à 8 ans après la naissance et ils ont été toujours exempts de symptômes.

5) Traitement au Salvarsan pendant la grossesse avec ou sans traitement mercuriel antérieur. 
Nous avons examiné 79 mères syphilitiques qui donnèrent naissance ḋ 19 enfants syphilitiques et $\dot{a} 60$ enfants sains. La différence avec les cas traités uniquement au mercure est frappante. Cependant, cette différence est probablement un peu moindre que les chiffres semblent l'indiquer. 23 des malades proviennent, en effet, de la clientèle privée ou de celle de la policlinique et toutes ces malades, outre le traitement au Salvarsan, ont reçu un traitement mercuriel très soigneux et très intense. Ces 23 malades ont toutes donné naissance à des enfants sains. Même en déduisant ces malades, chose qu'évidemment on ne peut pas faire, parce que dans ce cas il faudrait aussi déduire du groupe précedent toutes les malades qui ont été particulièrement bien traitées au mercure, la différence demeure cependant imposante. Comme tous les enfants sains ont été suivis pendant une période variant de 6 mois à 6 ans et qu'on peut indubitablement admettre qu'ils ont échappé à l'infection, il en résulte avec une absolue certitude que le trailement au Sa!varsan ajouté au traitement mercuriel habituel, pendant la grossesse a une importance primordiale pour l'enfant. Dans aucune des statistiques publiées jusqu'à ce jour, on ne trouve des matériaux qui démontrent cela aussi sûrement que les nôtres, car, tous les enfants d'apparence saine n'avaient été observés que pendant un temps très court après la naissance. On doit donc dire d'une manière absolue, d'après ce résultat, que le médecin qui néglige de traiter par le Salvarsan une femme enceinte encourt une grave responsabilité.

En ce qui concerne le traitement employé, toutes les malades, à l'exception d'une seule, ont reçu du mercure et du Salvarsan; le mercure soit sous forme de frictions ou d'injections de sels insolubles, et le Salvarsan, soit sous forme de Salvarsan ancien, de Néosalvarsan, soit sous forme de Salvarsan argentique, soit enfin sous forme de Galyl. Le résultat des divers modes de traitement au Salvarsan ressort du petit tableau suivant:

TABLEAU XIV.

$\begin{array}{lrccc} & 606 & 914 . & \text { Salvarsan argentique. } & \text { Galyl. } \\ \text { Enfants sains } & 24 & 19 & 16 & 1 \\ \text { Enfants syphilitiques } & 8 & 11 & 0 & 0\end{array}$


D'après ce tableau, le Néosalvarsan aurait une action relativement défavorable; le Salvarsan argentique est celui qui agirait le mieux. Nous croyons cependant qu'il ne faut pas attribuer une trop grande importance à ces chiffres.

En effet, les malades traitées au Néosalvarsan sont toutes des malades de l'hôpital qui, d'une manière générale, n'ont reçu qu'un seul traitement au mercure et au Salvarsan, tandis que 15 des 16 malades traitées au Salvarsan argentique proviennent de notre clientèle particulière ou de celle de nos consultations, et comme nous l'avons mentionné, ces malades ont reçu également un traitement intensif au mercure. Comme ces malades traitées au Salvarsan argentique ont été aussi traitées d'une manière plus intensive que la majorité des autres, ce résultat si favorable au Salvarsan argentique, ne peut pas être, sans plus, attribué à ce produit en tant que tel. Mais, en tout cas, le résultat ne parle pas contre le Salvarsan argentique. Ce qui a été dit des 15 malades traitées au Salvarsan argentique s'applique aussi à 8 des malades traitées à l'Altsalvarsan. Celles-ci, en plus du traitement au Salvarsan, avaient reçu un traitement mercuriel intensif et toutes donnèrent naissance à des enfants sains. Une seule malade de l'hôpital Rudolph. Bergh n'avait reçu que 10 injections de Salvarsan argentique, mais elle n'avait pas eu simultanément de traitement mercuriel; elle avait une manifestation secondaire généralisée. Son enfant demeura sain pendant 6 mois; mais à cette époque, il mourut à la suite d'une bronchopneumonie. L'autopsie fut pratiquée et on ne trouva pas d'altérations syphilitiques; immédiatement avant la mort, cet enfant avait une réaction de Wassermann négative.

13 de nos malades avaient été auparavant traitées au mercure; elles ne reçurent de Salvarsan qqu'au cours de la grossesse. Elles donnèrent naissance à 10 enfants sains et à 3 syphilitiques, nombres dont les rapports réciproques sont à peu près comme 19 est à 60 . On n'a pas constaté, non plus, que l'ancienneté de la maladie ait joué un rôle tant soit peu important. Neuf malades dont la maladie remontait entre 4 et 10 ans donnèrent naissance à 2 enfants syphilitiques (en 5 ans) et à 7 enfants sains (en 4 ans) et à 1 en 8 ans et 1 en 10 ans.

Dans aucun des cas il n'y eut de syphilis post-conceptionnelle.

La nature de la maladie ressort du tableau ci-dessous. 
TABLEAU XV.

\begin{tabular}{cccccc} 
Nature de la maladie & \multicolumn{3}{c}{ Nombre de cas } & $\begin{array}{c}\text { Enfants } \\
\text { syphilitiques }\end{array}$ & Enfants sains \\
Syphilis primaire & $\ldots$ & 1 & 0 & 0 & 1 \\
Syphilis secondaire & $\ldots$ & 24 & 9 & 15 \\
Syphilis tertiaire $\ldots \ldots$ & 1 & 0 & 1 \\
Syphilis latente & $\ldots \ldots$ & 53 & 10 & 43
\end{tabular}

Il ressort de ce tableau que la syphilis latente donne un pronostic relativement meilleur pour les enfants que la syphilis secondaire, chose qui était, a priori, probable. Cependant, il faut faire remarquer à ce propos que 21 sur 23 de nos malades de la clientèle privée et de la clientèle de consultations figuraient parmi les syphilitiques latentes et 2 seulement parmi les syphilis secondaires, ce qui déplace un peu les nombres. Mais la syphilis latente, comme il est logique, est toujours plus fávorable que la secondaire.

La réaction de Wassermann fut pratiquée lors de l'accouchement chez 11 mères d'enfants syphilitiques. 2 mères avaient un Wassermann négatif; 9 en eurent une positive, avec l'intensité ci-dessous.

TABLEAU XVI.

\begin{tabular}{ccc}
$\begin{array}{r}\text { Dose inhibitrice } \\
\text { minimum en c. c. }\end{array}$ & \multicolumn{3}{c}{ Nombre } \\
0,2 & $\ldots \ldots \ldots \ldots \ldots \ldots$ & 4 \\
0,1 & $\ldots \ldots \ldots \ldots \ldots \ldots$ & 0 \\
0,05 & $\ldots \ldots \ldots \ldots \ldots \ldots \ldots$ & 1 \\
0,025 & $\ldots \ldots \ldots \ldots \ldots \ldots$ & 3 \\
0,01 & $\ldots \ldots \ldots \ldots \ldots \ldots \ldots$ & 1
\end{tabular}

Chez 60 mères d'enfants syphiliticues, la réaction de Wassermann a été pratiquée 39 fois; elle fut négative dans 36 cas et 3 fois positive (une fois avec 0,1 c. c. et 2 fois avec 0,05 ).

De même, on voit ici aussi que l'existence de la réaction de Wassermann chez la mère au moment de l'accouchement a une certaine importance pronostique.

En ce qui concerne la descendance syphilitique, nous eûmes 3 avortements après le 6:ème mois, 2 enfants macérés et 14 enfants 
vivants. Sur ces derniers, 11 avaient une réaction de Wassermann positive, 3 étaient sains en apparence avec Wassermann négatif; mais tous eurent des manifestations syphilitiques, respectivement 1 mois, 4 mois et 5 mois après la naissance. Les 60 enfants sains ont tous été suivis de 6 mois jusqu'à 6 ans après leur naissance; la majorité n'a pas été suivie pendant moins de deux ans.

La réaction de Wassermann a été dosée chez 9 enfants et elle avait l'intensité auivante:

TABLEAU XVII.

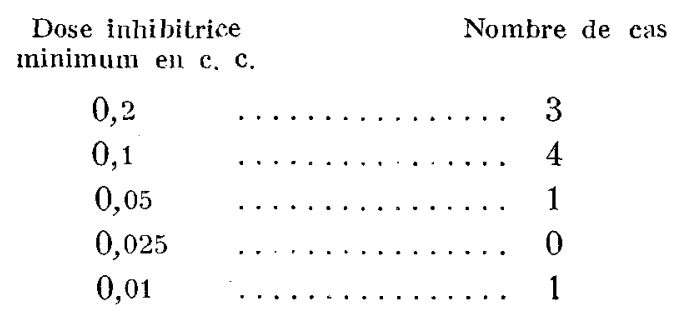

Nous arrivons maintenant à deux petits groupes particuliers.

6) Salvarsan administré avant la grossesse, mercure administré pendant la grossesse.

Nous avons examiné 26 mères syphilitiques; 7 donnèrent naissance à des enfants syphilitiques et 19 à des enfants sains. Le résultat est donc favorable, il est à peu près aussi favorable que dans le cas du Salvarsan administré pendant la grossesse. La nature du traitement antériur au Salvarsan ressort du tableau suivant.

\section{TABLEAU XVIII.}

$\begin{array}{lrrc} & 606 & 914 . & \text { Salvarsan argentique } \\ \text { Enfants sains } & 12 & 6 & 1 \\ \text { Enfants syphilit. } & 4 & 3 & 0\end{array}$

Il est évident que ces chiffres sont trop peu considérables pour permettre de tirer des conclusions décisives. La traitement originellement employé consistait dans tous les cas dans un traitement mixte au Salvarsan et au mercure; pendant la grossesse on employa pour ainsi dire dans tous les cas, le traitement par frictions. 
Le premier traitement au Salvarsan et au mercure fut fait lors de la première manifestation syphilitique secondaire; dans trois cas seulement, il s'agissait de l'accident initial; ces trois femmes donnèrent naissance à des enfants sains.

Pendant la grossesse, dans tous les cas, sauf un, il s'agissait de syphilis latente; l'une des malades avait des manifestations secondaires récidivantes: néanmoins, elle eut un enfant sain. La réaction de Wassermann fut pratiquée chez 6 mères qui avaient eu des enfants syphilitiques, elle fut négative dans deux cas et positive dans 4 ( 2 fois avec 0,2 cc. 1 fois avec 0,1 et 1 fois avec 0,05 ).

Chez 12 mères qui eurent des enfants sains, elle fut négative 12 fois. La descendance des syphilitiques fut la suivante: 2 avortements, 2 foetus tout à fait macérés, 3 fois des enfants vivants dont deux eurent des manifestations immédiatement au moment de la naissance; un des enfants était sain en apparence au moment de l'accouchement et il avait un Wassermann négatif; ce n'est qu'un mois plus tard qu'il eut des manifestations et une réaction positive.

Les 19 enfants sains furent observés de 6 mois à 3 ans après la naissance.

7) Salvarsan avant la grossesse; Salvarsan pendant la grossesse.

Nous avons examiné 7 mères syphilitiques; 6 eurent des enfants sains, une eut un enfant syphilitique en dépit de tout traitement. Cette dernière avait toutes les deux fois reçu de l'Altsalvarsan. Les autres. avaient reçu toutes les combinaisons imaginables d'Altsalvarsan, de Néosalvarsan et de Salvarsan argentique; de telle manière qu'il n'est pas possible de tirer des conclusions déterminées de ces résultats.

L'enfant syphilitique était né dans la deuxième année de la maladie; les 6 enfants sains naquirent entre 2 et 6 ans de la maladie. Toutes les malades avaient une syphilis latente au cours de leur grossesse et, au début, on les avait soumises au traitement lors de l'apparition complète des manifestations secondaires.

Chez toutes les mères, le. Wassermann ètait négatif, de même chez la malade qui donna naissance à l'enfant syphilitique.

Le seul enfant qui eut la syphilis, avait déjà des manifestations étendues lors de la naissance. La réaction de Wassermann était $0.0 .020,100$; les 6 enfants sains furent tous suivis pendant une periode allant de 1 an à 5 ans après leur naissance. 
Le résultat d'ensemble des recherches précédentes est donc que: les mères dont la syphilis n'a pas été traitée ont presque toujours des enfants syphilitiques et, en outre que, un traitement au Salvarsan pendant la grossesse donne de bien meilleurs résultats qu'un traitement mercuriel. Tout cas de syphilis pendant la grossesse doit donc être traité au Salvarsan, pourvu, bien entendu, que la malade puisse le supporter. Il faut, en effet, se souvenir qu'il n'est pas rare que les reins soient atteints vers la fin de la grossesse. Un examen soigneux de l'urine qui, cela va sans dire, sera fait avant tout traitement au Salvarsan, est ici particulièrement indiqué. Si l'on constate que les reins sont atteints, il faut attendre jusqu'à ce que l'urine soit redevenue normale et la diurèse également. Si la femme enceinte, se porte bien sous les autres rapports, le traitement au Salvarsan est très bien toléré. Toutes les malades que nous avons traitées nous-mêmes dans notre clientèle privée ou de consultations l'ont parfaitement toléré. Si nous nous demandons maintenant comment nous devons conduire le traitement pendant la grossesse, la réponse variera un peu suivant qu'il s'agit d'une première manifestation syphilitique ou d'un cas de syphilis latente. Une première manifestation qui, par exemple, est traitée au début de la grossesse, devra d'abord recevoir pendant une semaine un traitement mercuriel préparatoire, par exemple 7 frictions à $3 \mathrm{gr}$.; puis on administrera le Salvarsan, en même temps que le traitement mercuriel sera continué. On donnera en tout 4 injections de Salvarsan à une semaine d'intervalle; le traitement par les frictions est continué, jusqu'à ce que la malade ait reçu 50 frictions au total. La question de savoir la préparation de Salvarsan à employer est peut-être moins importante. D'après ce que GENNERICH a constaté dans le traitement abortif, nous donnerions la préférence l'Altsalvarsan ou au Salvarsan argentique; le premier serait donné à des doses de $30,40,40$ et 40 centigr.; le second à la dose de $15,20,25$, et 30 centigr: pour tous les deux, la dose varierait suivant le poids de la malade et son état général. Après ce traitement, repos d'un mois; puis nous donnerons une nouvelle cure de frictions ( 30 frictions). Nouveau repos d'un mois; nous voici maintenant au milieu de la grossesse; nous recommanderons à ce moment de donner un traitement mixte au Salvarsan et au mercure. Comme, d'après les remarquables recherches de Oluf Thomsen, c'est précisément au milieu de la grossesse que les 
spirochètes commencent à émigrer à travers le placenta pour se rendre vers le foetus et que le pronostic est d'autant plus mauvais que cette migration transplacentaire est plus précoce il est indiqué d'administrer juste à ce moment un traitement énergique. Nous reprenons donc le traitement au Salvarsan et au mercure décrit plus haut. Après celui ci, repos d'un mois; puis nous donnons de nouveau 30 frictions à $3 \mathrm{gr}$. Nouveau repos d' un mois; nous sommes maintenant tout proches de l'accouchement; peut-être pouvonsnous pendant cette période donner quelques frictions. C'est ainsi que pendant les 5 dérnières années écoulées, nous avons traité toutes nos malades de la clientèle particulière et des consultations et toutes ces malades ont parfaitement supporté le traitement et toutes ont donné naissance à des enfants sains.

Une femme doit toujours recevoir un traitement pendant sa grossesse quelque soit le moment de la maladie auquel elle se trouve. Ainsi qu'il ressort de nos cas, la maladie peut être transmise au foetus même 20 ans après l'infection de la mère; il faut donc que toute femme syphilitique soit traitée pendant sa grossesse quelle que soit l'ancienneté de sa maladie.

Même quand une femme a reçu le traitement le plus soigneusement exécuté pendant les premières années de sa maladie, elle peut ultérieurement donner naissance à des enfants syphilitiques, si elle n'est pas traitée au cours de sa grossesse. Nous avons déjà mentionné un exemple typique de ce cas; une jeune femme est contaminée de syphilis; on lui administre d'abord du Salvarsan et du mercure et ensuite un traitement mercuriel soigneusement exécuté pendant 3 ans. 4 ans après l'infection, elle devient enceinte; on ne lui donne pas d'autre traitement antisyphilitique que le traitement si bien exécuté qu'elle avait déjà reçu et elle accouche d'un foetus macéré avec des lésions syphilitiques et une réaction de Wassermann fortement positive.

En aucune circonstance, on ne doit se fier à une réaction de Wassermann négative pendant la grossesse. La malade doit être traitée tout de même. Nous citerons un exemple typique: à l'hôpital Rudolph Bergh, une malade avait reçu un traitement abortif qui semblait avoir bien réussi; ce traitement était dirigé contre un accident secondaire récent (la première roséole). On la suivit pendant un an après le traitement; elle avait constamment un Wassermann négatif 
et pas de symptômes cliniques. Elle devint alors enceinte; à cette date nous étions enclins à surestimer la valeur du Salvarsan; nous examinâmes la malade chaque mois pendant sa grossesse; elle était toujours sans aucun symptôme clinique et sérologique. Quinze jours avant l'accouchement, elle eut une manifestation et elle mit au monde un enfant avec intense manifestation de syphilis congénitale. Ce cas peut être complété par plusieurs analogues.

Donc: toute femme syphilitique doit, pendant sa grossesse, être traitée par le Salvarsan et le mercure, peu importe le moment de la maladie auquel elle se trouve, sans tenir compte non plus du traitement antérieur et sans tenir compte aussi du résultal de la réaction de Wassermann.

En terminant, nous devons mentionner brièvement quelques points importants, à l'élucidation desquels nos matériaux abondants fournissent une remarquable contribution. Le premier c'est:

Importance de l'ancienneté de l'affection pour la transmission de l'infection à la descendance. Comme on le sait, Kassowitz a autrefois établi un schéma cité partout, suivant lequel les femmes syphilitiques, au début de leur maladie, ont avortement sur avortement; puis vient l'accouchement prématuré avec foetus macérés: puis accouchement prématuré d'enfants syphilitiques vivants, qui succombent peu après leur naissance; ensuite, naissance prématurée d'enfants vivants qui présentent bientôt des manifestations syphilitiques et plus tard naissance à terme d'enfants qui n'ont de manifestations syphilitiques qu'au bout d'un certain temps et, enfin, naissance à terme d'enfants tout à fait sains. Cette diminution spontanée de la transmissibilité au foetus, mentionnée dans tous les Traités sur la syphilis, doit être acceptée avec réserves. Il est indubitable que cette diminution peut se rencontrer, mais on trouve un nombre considérable de cas où elle n'existe pas. Déjà, en 1912, Marcus attirait l'attention là dessus, car il faisait ressortir que c'était le traitement de la maladie pendant la grossesse qui importait davantage que son ancienneté. RöNne ET BoAs, en 1915, examinant 33 familles avec enfants syphilitiques ont constaté que ce schéma ne s'applique pas dans un grand nombre de cas. De même, Rasch, dans sa Monographie sur la syphilis dans le Traité de Médecine interne écrit que la transmission de la syphilis à la descendance se fait d'une manière très capricieuse. En parcourant nos matériaux 
considérables, on voit que, nulle part, ce schéma ne se montre dans sa pureté classique. Quand les malades n'ont pas été traitées pendant la grossesse, les enfants sains, les avortements et les enfants syphilitiques alternent. Nous allons en citer quelques exemples concrets:

Une jeune femme contracte la syphilis en 1909; elle est enceinte en même temps et elle est énergiquement traitée au mercure pendant sa grossesse. Elle n'eut jamais d'autre traitement. Elle a 4 enfants:

1) 1909. Enfant sain.

2) 1910. Enfant mort en bas âge (de syphilis?)

3) 1912. Enfant sain.

4) 1917. Enfant avec manifestations syphilitiques et réaction de Wassermann positive.

Un exemple encore plus éclatant est le suivant:

Syphilis en 1891-1892; traitée par 50 frictions à 5 gr. Pas d'autre traitement ultérieur.

1) 1895. Mort-né.

2) 1897. Enfant sain (Wassermann négatif.) (15 ans)

3) 1900. Enfant mort de syphilis.

4) 1902. Accouchement prématuré (Mort-né)

5) 1903. Avortement

6) 1904. Enfant sain (Wassermann négatif) (8 ans)

7) 1905. Enfant sain (Wassermann négatif) (7 ans)

8) 1906. Enfant mort en bas âge (de syphilis?)

9) 1907. Avortement.

10) 1908. Enfant sain (Wassermann négatif) (4 ans)

11) 1909. Enfant mort en bas âge (de syphilis?)

12) 1912. Enfant mort de syphilis congénitale.

Il est difficile de s'imaginer quelque chose de moins harmonique et de plus irrégulier. On portera tout spécialement son attention sur le fait que deux enfants sains nés en 1904 et en 1905 sont suivis de toute une série d'enfants syphilitiques; nous n'avons pas ainsi la moindre garantie que les enfants à venir seront également sains, ainsi que le disent les anciens traités de syphiligraphie.

Tant que la mère se trouvera à l'âge où elle est capable d'avoir des enfants, on pourra voir des cas de syphilis congénitale dans sa descendance. Le tableau suivant présente le nombre d'enfants 
syphilitiques et sains après la troisième année de ta contamination quand la mère n'est pas traitée au cours de la grossesse. Dans les cas de ce tableau, il s'agit de mères qui dans la ou les premières années après l'infection ont été plus ou moins bien traitées au mercure ou au Salvarsan, mais qui n'ont pas été traitées pendant la grossesse. Quand il s'agit de mères qui n'ont pas été du tout traitées, on ne peut presque jamais déterminer nême d'une façon approximativement certaine l'ancienneté de l'infection.

TABLEAU XIX.

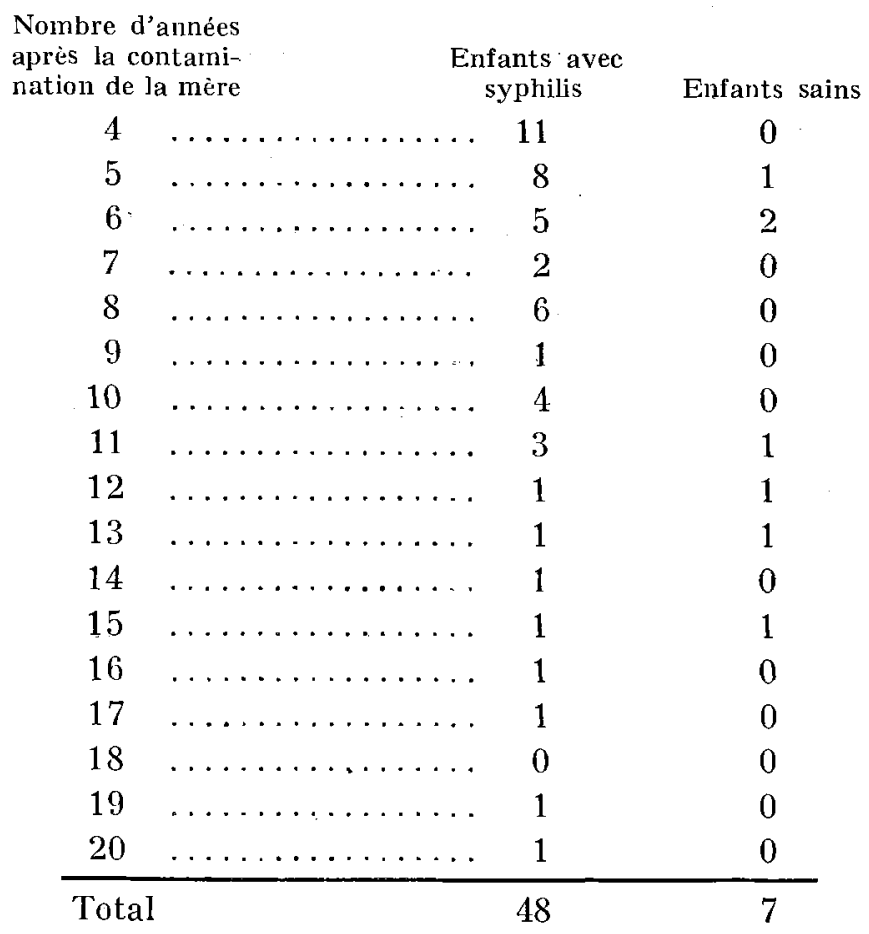

On voit donc que lorsqu'une mère syphilitique devient enceinte entre la 4:ème et la 20:ème années après l'infection, s'il n'y a eu aucun traitement pendant la grossesse, elle aura beaucoup plus d'enfants syphilitiques que d'enfants sains (48 syphilitiques, 7 sains), ce qui n'indique pas que la capacité de transmettre l'infection diminue avec l'ancienneté de celle-ci. Une série parallèle de mères syphilitiques dans les 3 premières années de l'infection montre 42 enfants sy- 
philitiques et 5 enfants sains. Le pourcentage d'enfants syphilitiques et d'enfants sains dans ces deux séries est presque égal; il y a seulement une très minime prédominance d'enfants syphilitiques dans la dernière série. Quoiqu'il en soit, une diminution importante de la transmissibilité à la descendance au fur et à mesure que la maladie devient plus ancienne, ne ressort pas du tout de nos chiffres. D'autres auteurs ont vu des cas encore plus tardifs de transmission de la syphilis. Marcus en a publié deux dans lesquels la syphilis de la mère remontait à 24 et 26 ans et W. Boeck en a publié un autre dans lequel la syphilis de la mère avait 37 ans de date (!); la mère avait été infectée dans son enfance.

La question est tout à fait différente quand l'affection de la mère est traitée pendant la grossesse. C'est beaucoup plus le traitement que l'ancienneté de la maladie qui importe ici (Marcus). Ainsi que l'a déjà dit Fournier, on peut, chez une femme syphilitique, volontairement déterminer, ou tout au moins dans la majorité des cas, que son enfant sera sain ou syphilitique, suivant que l'on aura traité la mère pendant la grossesse ou qu'on aura négligé de le faire. Un de nos cas en fournit un exemple très frappant. Une femme est infectée, probablement au môment même de la conception. Elle n'est pas traitée et accouche d'un enfant syphilitique macéré. Peu après, elle est de nouveau enceinte et cette fois on la traite au Néosalvarsan et au mercure; elle accouche alors d'un enfant sain qui continue à demeurer en bonne santé. La femme suspend alors tout traitement; elle devient enceinte de nouveau un an et demi après; elle ne reçoit pas du tout de traitement pendant sa grossesse et elle accouche cette fois-ci encore d'un enfant mort et macéré. Pendant une nouvelle grossesse survenue un an après, elle est traitée et cette fois par un traitement mercuriel intensif et elle accouche d'un enfant sain. Nous pourrions citer beaucoup de cas semblables à celui-ci.

Comment se comporte la réaction de Wassermann dans la syphilis congénitale. Nous ne perdrons pas dans les détails sur cette question, car nos résultats confirment tout à fait ce qu'ont antérieurement publié Thomsen et BOAs; cependant leurs résultats sont corroborés par nos matériaux qui sont beaucoup plus considérables que les leurs. Tout d'abord, Thomsen et BoAs ont trouvé, ainsi que nous l'avons mentionné plusieurs fois, que la réaction de Wasser24 - Acta Gynecologica. Vol. I. Fasc. 3. 
mann chez la mère au moment de l'accouchement a une certaine importance pronostique, car les enfants de mères réagissant positivement lors de l'accouchement, sont bien plus souvent atteints de syphilis que ceux dont les mères ont une réaction négative au moment de l'accouchement. Nos résultats actuels ressortent du tableau ci-joint

TABLEAU XX.

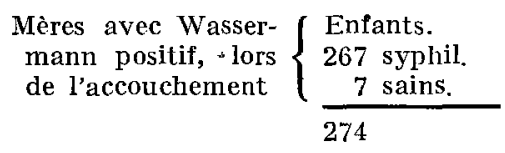

$$
\begin{aligned}
& \text { Mères avec Wasser- Enfants. } \\
& \text { mann négatif, lors }\{24 \text { syphil. } \\
& \text { de l'accouchement } \\
& 80 \text { sains. } \\
& 104
\end{aligned}
$$

Si, immédiatement avant l'accouchement, il y a eu un traitement spécifique, la réaction négative n'a qu'une minime valeur pronostique.

En outre, Thomsen et Boas, ainsi que nous l'avons mentionné en passant, ont trouvé que, dans des cas très rares, les enfants peuvent avoir une réaction positive à la naissance, sans présenter par la suite des symptômes de syphilis. Il est très probable qu'il s'agit là du passage au foetus de substances réagissantes provenant du sang de la mère.

Nous avons vu trois de ces cas, tous chez des mères traitées au mercure. Les réactions étaient positives chez les enfants avec 0,2 c. c. comme quantité minimum et positives chez les mères avec 0,1 comme quantité minimum, dans deux cas et avec 0,05 dans un cas. Chez ces trois enfants, la réaction disparut dans l'espace de 14 jours et aucun de ces enfants pendant la période d'observation qui dura 3 mois ne présenta de signes de syphilis. Comme ces enfants ont, par la suite, échappé à notre observation, nous n'avons pas pu les faire entrer dans le total de nos résultats; mais, en raison de l'importance pratique considérable de la question, nous n'avons pas voulu négliger d'en parler ici.

Thomsen et Boas ont, en outre, constaté que les mères d'enfants syphilitiques sont toujours elles-mêmes syphilitiques, car 11 mères qui donnèrent naissance à des enfants syphilitiques, sans présenter elles-mêmes des signes objectifs ou des commémoratifs de syphilis, eurent toutes une réaction positive. L'observation bien connue de Colles qu'un enfant atteint de syphilis congénitale ne contagionne 
jamais sa mère, s'explique ainsi facilement. Dans nos matériaux, il existait 77 fois des enfants syphilitiques sans que la mère ait rien soupçonné de l'infection ou en ait présenté quelque signe. 75 fois, les mères avaient une réaction de Wassermann positive; deux fois seulement la réaction fut négative chez elles; mais l'une d'elles était positive avec 0,4 c. c. 'On est en droit d'admettre que, dans ces deux cas, la réaction a échoué (on peut aussi voir, rarement il est vrai, une réaction de Wassermann négative dans la syphilis secondaire) ou que les mères avaient été traitées, malgré leurs dénégations. Il nous parait très invraisemblable d'admettre qu'il se soit agi d'infection paternelle. Si nous avions pu réussir à suivre ces deux mères pendant un temps assez long, certainement nous aurions trouvé chez elles à un moment ou à un autre, une réaction positive, ou bien elles auraient présenté des symptômes cliniques de syphilis.

En terminant, nous reconnaitrons bien qu'il y a de nombreux côtés du problème "Syphilis et Grossesse» dont nous n'avons pu nous occuper.

Tout d'abord, cela s'applique à la question du diagnostic et surtout aux renseignements que peuvent nous fournir les altérations syphilitiques du cordon ombilical et du placenta et en ce qui concerne les enfants morts, les altérations du foie, de la rate, du pancréas, du système osseux, toutes questions sur lesquelles le grand travail, malheureusement trop peu connu, d'Oluf THomsen fournit d'excellentes données.

Le traitement de la syphilis pendant la grossesse a une importance sociale considérable et il faut l'aborder par toutes les voies possibles.

L'importance d'une institution, telle que la consultation pour femmes enceintes indigentes, établie à la maternité de Rigshospital, se manifeste en ce que, on pourra, ainsi, déceler la syphilis à une période précoce de la grossesse et qu'on sera, ainsi, capable de soumettre les mères à un traitement anti-syphilitique réellement efficace.

Nous sommes heureux, pour terminer, de remercier les divers médecins-chefs (Prof. Rasch, Prof. Ehlers, Dr Ludvig Nielsen et Dr JersiLd) de la grande amobilité avec laquelle ils ont mis à 
notre disposition les renseignements fournis par les registres d'observation, sans lesquels il nous eut été impossible de mener à bonne fin le présent travail.

\section{Bibliographie.}

Adams, J.: Brit. med. journal. Nr. 3096, 1920.

Ab ams, J.: Lancet. Nr. 5072. 1920.

BaIsch: Monatschr. f. Geb. und Gyn. Bd. 34.

BAR, P.: Soc. d'obstét. de Paris. Séance 16/2 - 1911.

BAR et DaunaY: L'obstétrique 1909.

Beck, A. C.: Americ. journal of obst. and gyn. Vol. 2. Page 416.

BjorkenHeim, E.: Ergeb. der Geb. und Gyn. Bd. 3.

Boas: Die Wassermann'sche Reaktion. Troisième édition. Berlin 1922.

Brisson: Thèse de Paris, 1913.

Bunzel, E.: Wiener klin. Wochenschr. Nr. 36, 1909.

EhLERs, E.: Om Dødeligheden ved medfødt Syphilis hos Børn. 1914.

Fabre et Bourret: Presse médicale. Nr. 97. 1912.

Findeay, L.: Brit. med. journal. Nr. 3110. 1920.

Findlay, L.: Dubl. journal of med. science. Nr. 5. 1920.

Findeay, L.: Brit. med. journal. Nr. 3178. 1921.

Frankl, O.: Monatschr. f. Geb. und Gyn. Bd. 31.

Fullerton, W. D.: American journal of obst. Bd. 74.

Goodman, H.: Surgery, Gyn. and Obstetrics. Bd. 30. 1920.

Goudy, L.: Thèse de Bordeaux. 1908.

Harman, B.: Cité d'après Creadick.

HENDRY, R. A.: Lancet. Nr. 5072. 1920.

HENDRY, R. A.: Brit. med. journal. Nr. 3125. 1920.

Holth, M.: Deutsche med. Wochenschr. Nr. 10. 1913.

HorNung, R.: Ergeb. der Geb. und Gyn. Bd. 8. 1920.

HÜFFEL: Monatschr. f. Geb. und Gyn. Bd. 34. 1911.

JAEGER, O.: Cité d'après le Zentralblatt f. Gyn. Nr. 1. 1912.

JAEGER, O.: Münch. med. Wochenschr. Nr. 35. 1910.

Jeannin, M. C.: Presse médicale. Nr. 2. 1912.

JEANS: Americ. journ. of disease of children. 1916.

Jeanselme: Annales de gyn. et obst. Année 40. 1913.

Kaufmann-Wolf und Abrahamson: Zeitschr. f. klin. Medicin. Bd 89. 1920.

LAнм, W.: Arch. f. Gyn. Bd. 112.

LANGe, E.: Med. Klinik. Nr. 20. 1911.

Lemeland et Brisson: Annales de Gyn. et Obst. 2 S. Tome 10. 1913.

Lobenstein, R. W.: Americ. journal of obstr. Vol, 69. 1914.

Loeb: Monatschr. f. Geb. und Gyn. Bd. 50.

Loeser, A.: Berliner klin. Wochenschr. Nr. 42. 1920. 
Loeser, A.: Cité d'après le Zentralblatt f. Gyn. Nr. 44. 1920.

MARkus: Klinische Beobachtungen über die Prognose der kongenitalen Syphilis. 1912. Archiv f. D. u. S. Kr.

Meyer, E.: Zeitschr. f. Geb. und Gyn. Bd. 77. 1915.

Opitz, E.: Med. Klinik. Nr. 30. 1908.

Peiser: Berliner klin. Wochenschr. Nr. 1. 1911.

Reuben Peterson: Surgery, Gyn. and Obstetrics. V. 23. 1916.

Placzen: Leipzig. 1918.

Reiche, A.: Zeitschr. f. Kinderheilkunde. Bd. 12. 1915.

RUdAuX et Le Lorier: Annales de gyn. 1911.

SaEnger, H.: Monatsclar. f. Geb. und Gyn. Bd. 46.

Sauvage, C.: Annales de gyn. et obst. Année 40: 1913.

Schwab: Zentralblatt f. Gyn. Nr. 13, 1920.

Stangenberg, J.: Zentralblatt f. Gyn. Nr. 28. 1920.

Steinert und Fischl: Arch. f. Kinderheilkunde. Bd. 95. 1920.

Steinert und Fischl: Arch. f. Kinderheilkunde. Bd. 96. 1921.

Stühmer und Dreyer: Zeitschr. f. Geb. und Gyn. Bd. 84. 1921.

Thomsen: Studier over de af den medfödde Syfilis hos Fosteret og det nyfødde Barn foraarsagede patologisk-anatomiske Forandringer. Copenhague 1912.

Thomsen \& Boas: Arch. f. Derm. u. Syph. Bd. 111.

Trinchese, J.: Hegars Beiträge. Bd. 18. 1913.

Watter, J. G. Mc: Brit. med journal. Nr. 3103. 1920.

Weghelmani: Deutsche med. Wochenschr. Nr. 15. 1909.

Williams, W.: Bulletin. John Hopkins Hospital. Nr. 356. 1920.

Williams, W.: Americ. journal of obst. and gyn. Vol. 1. 1920.

Pour la bibliographie récente, nous unvoyons à A. N. CREAdick: Antenatal syphilis. Collective review dans The american Journal of obstetrics and gynæcology Vol. 2. N:o 4 octobre 1921. On y trouve une liste bibliographique de la littérature postérieure à 1916 avec 129 travaux et renvois aux ouvrages d'ensemble, renfermant la littérature antérieure à 1916. 